\title{
Robustness of attractors in tapping mode atomic force microscopy
}

\author{
Abhilash Chandrashekar • Pierpaolo Belardinelli • Urs Staufer • Farbod Alijani $($ C
}

Received: 26 October 2018 / Accepted: 30 May 2019 / Published online: 13 June 2019

(C) The Author(s) 2019

\begin{abstract}
In this work, we perform a comprehensive analysis of the robustness of attractors in tapping mode atomic force microscopy. The numerical model is based on cantilever dynamics driven in the Lennard-Jones potential. Pseudo-arc-length continuation and basins of attraction are utilized to obtain the frequency response and dynamical integrity of the attractors. The global bifurcation and response scenario maps for the system are developed by incorporating several local bifurcation loci in the excitation parameter space. Moreover, the map delineates various escape thresholds for different attractors present in the system. Our work unveils the properties of the cantilever oscillation in proximity to the sample surface, which is governed by the so-called in-contact attractor. The robustness of this attractor against operating parameters is quantified by means of integrity profiles. Our work provides a unique view into global dynamics in tapping mode atomic force microscopy and helps establishing an extended topological view of the system.
\end{abstract}

Keywords Atomic force microscopy · Tapping mode Basins of attraction - Dynamical integrity - Bifurcation chart $\cdot$ Basin erosion · Integrity profiles $\cdot$ In-contact attractor $\cdot$ Robustness

A. Chandrashekar · P. Belardinelli · U. Staufer .

F. Alijani $(\varangle)$

Precision and Microsystems Engineering, TU Delft,

Mekelweg 2, 2628 CD Delft, The Netherlands

e-mail: F.Alijani@tudelft.nl

\section{Introduction}

Atomic force microscopy (AFM) has become one of the most prominent characterization tools in modern science. It is ubiquitously used to characterize and manipulate surface properties of materials down to atomic resolution in both air and liquid environments [1]. Among the various AFM operational modes, tapping mode AFM (TM-AFM) [2] also known as amplitude modulation AFM (AM-AFM) [3] is one of the most extensively used techniques to obtain high-resolution images of wide variety of samples. Owing to reduced lateral forces and high-phase sensitivity, TM-AFM is widely popular in soft matter [4], biological samples [5] and polymer [6] applications.

TM-AFM is based on the near-resonant excitation of a microcantilever with a sharp tip at its free end that is vibrating in the vicinity of the sample. The vibrations in the cantilever are influenced by the tip-sample interaction forces, which modifies the beam dynamics. In general, the tip-sample interaction is nonlinear and comprises long-range attractive Van der Waals forces, shortrange quantum mechanical repulsive forces, adhesive and contact forces. The nonlinear response due to tipsample interaction is even more involved in the presence of electrostatic and capillary forces [7]. In the existence of such complex nonlinearities, a comprehensive understanding of the multi-stable response is crucial, since these nonlinearities can be efficiently utilized for extracting several nanomechanical properties of the sample $[3,8-10]$. Furthermore, the accuracy of 
imaging and nanomechanical characterization of sample surfaces from measured data depends crucially on the deconvolution of data with appropriate models.

In recent years, the underlying dynamics of AFM cantilever and its exploitation have been investigated by many authors [11-18]. The vast majority of these studies have been dedicated to study the microcantilever dynamics by utilizing a simplified single-degree-offreedom point mass model [16-18]. In this approach, first a static analysis is performed to determine the cantilever stiffness and then the equivalent mass is calculated based on the experimentally evaluated fundamental resonance frequency. Furthermore, the excitation is modelled as an external force acting on the point mass [16-18]. Such a lumped parameter model does not represent the conditions encountered in reality, where the microcantilever is subjected to base excitation at the clamped end by means of a piezoelectric actuator. The base excitation induces linear and nonlinear parametric excitations that are typically not captured by the lumped parameter model, thus failing to accurately describe the dynamics of the microcantilever $[13,14]$.

Continuous beam models on the contrary have proven to predict the nonlinear aspects of AFM cantilever dynamics accurately [12-15]. These models are able to provide precise and deeper insights into the physics behind the nonlinear phenomena such as amplitude jumps, period-doubling, and grazing bifurcations $[14,19]$. Therefore, in spite of the complexities involved in modelling of the AFM cantilever as a continuous beam, it allows for capturing the overall nonlinear aspects of the AFM dynamics [20].

In practical operation, the dynamics of AFM cantilever is influenced by several operating parameters such as tip radius, excitation amplitude, excitation frequency, and feedback values. The real-time variation of these parameters during an AFM operation can lead to unwanted dynamical phenomena such as bifurcations and unstable and aperiodic motions, which can decrease the reliability of results and strictly limit the operating ranges of the AFM. The dynamic models as well as the feedback strategies implemented in standard AFM systems tend to focus on governing the local dynamics of the system, leaving the impact on the global dynamics largely unknown. Therefore, in order to predict and control these dynamical events as well as preserving the stability of operation in TM-AFM, it is important to study and understand the nonlinear responses from a global perspective.
Currently, there are no detailed works on global dynamics of TM-AFM, which (i) evaluate the escape boundaries, (ii) estimate dynamical integrity, and (iii) perform detailed analysis of bifurcations. Existing literature has focused on the dynamical integrity and bifurcation scenarios of non-contact AFM [19,21]. But basins of attraction and erosion process of basin portraits in TM-AFM are lacking in the literature. The erosion of uncorrupted basins of attraction surrounding each main solution as a function of AFM operation parameters is of paramount importance from both theoretical and experimental perspective.

In this article, we elucidate the global dynamics and robustness of attractors in TM-AFM. Differently from existing works, which are based on limited analysis on the local dynamical behaviour, this work makes systematic use of bifurcation diagrams to highlight the appearance and disappearance of steady-state solutions. The latter offers an overall interpretation of the dynamic response with respect to operational parameters, namely excitation frequency and forcing amplitude. Additionally, we show that, by changing the operation parameters, a microcantilever initially in the primary resonant branch can escape from its local potential well and get captured by a second coexisting local potential well close to the sample surface. This regime of oscillation close to the sample surface, referred in this article as 'in-contact' attractor, is unexplored in the literature. In this work, we examine its evolution and robustness properties with frequency response curves and basins of attraction. Furthermore, the envelopes of local bifurcation boundaries are built to understand the escape scenarios of the solutions. Finally, integrity analysis is performed to quantify the steady-state solutions associated with all the attractors present in TMAFM.

Based on these motivations, the article is organized as follows: the modelling of the system is discussed briefly in Sect. 2. This is followed by a detailed analysis of frequency response of the system, bifurcation charts and response scenarios including escape of solutions in an excitation parameter space in Sect. 3. Particular attention is paid to the frequency response analysis of the in-contact attractor around its primary and parametric resonance. The results of this section are then utilized to build basins of attraction and dynamical integrity curves for the main attractors present in the system in Sect. 4. 


\section{Numerical model}

The classical beam theory, based on the Euler-Bernoulli assumptions, is used to obtain the continuous model for the AFM microcantilever shown in Fig. 1. The nomenclature used to describe the equations in this article is identical to the one described by Ruetzel et al. [12]. The deflection of the cantilever towards the sample is treated as positive, and the rest position of the cantilever is taken as reference. The considered microcantilever has a length $L$, mass density $\rho$, Young's modulus $E$, area moment of inertia $I$, and cross-sectional area $A$. The beam is clamped at $x=0$ and free at $x=L$. The tip-sample separation distance in the reference configuration is denoted by $Z$, and the total deflection of the microcantilever $w(x, t)$ can be expressed as $w(x, t)=u(x, t)+w^{*}(x)+y(t)$, where $u(x, t)$ is the deflection of the microcantilever relative to a noninertial reference frame attached to the base and $w^{*}(x)$ is the static deflection towards the sample due to tipsample interaction. The base excitation is generated by a dither piezo and is assumed to be harmonic, i.e. $y(t)=Y \sin (\Omega t)$, where $Y$ and $\Omega$ are the amplitude and frequency of excitation, respectively.

\subsection{Tip-sample interaction}

In TM-AFM, the microcantilever oscillates in close proximity to the sample surface. In our work, we use the Lennard-Jones (LJ) potential to describe the tipsample interactions [22]. Although the model doesn't take into account the real contact mechanics encountered in TM-AFM, it represents a generic tip-surface interaction potential which mimics qualitatively, the more detailed and computationally expensive models [13]. The LJ potential models the non-retarded dispersive Van der Waals forces as well as the short-

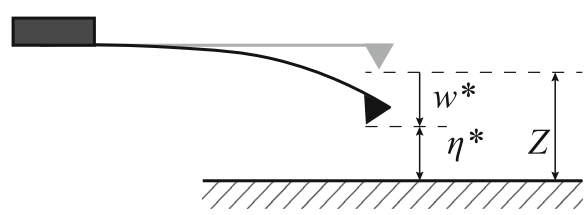

(a) range repulsive exchange interactions between two molecules. Assuming a spherical tip apex with radius $R$ and a flat sample surface, the interaction potential and the force are:

$U_{\mathrm{LJ}}=\frac{A_{1} R}{1260 z^{7}}-\frac{A_{2} R}{6 z}$,

$P_{\mathrm{LJ}}=-\frac{\partial U}{\partial z}=\frac{A_{1} R}{180 z^{8}}-\frac{A_{2} R}{6 z^{2}}$,

where $z$ is the instantaneous tip-sample separation gap. $A_{1}$ and $A_{2}$ are the Hamaker constants for the repulsive and attractive potentials, respectively. A positive interaction force implies repulsion. The Hamaker constants are $A_{1}=\pi^{2} \rho_{1} \rho_{2} \mathrm{c}_{1}$ and $A_{2}=\pi^{2} \rho_{1} \rho_{2} \mathrm{c}_{2}$, where $\rho_{1}$ and $\rho_{2}$ are the number densities of molecules in the interacting media and $c_{1}$ and $c_{2}$ are the interaction coefficients of intermolecular pair potential [22].

\subsection{Equation of motion}

The nonlinear static deflection of the microcantilever in the absence of base excitation is computed by solving for the equilibrium gap between the tip and the sample shown in Fig. 1. The static equilibrium gap $\eta^{*}$ at the free end is calculated as a function of the approach distance $Z$ through static balancing of the cantilever restoring force and the tip-sample interaction forces.

The dynamic equation of motion of the tip deflection $u(x, t)$ about its nonlinear equilibrium subjected to base harmonic motion is then derived through a single mode discretization of the Euler-Bernoulli beam equation. The interaction forces given by Eq. (1b) are assumed to be acting on the free end of the cantilever and are mathematically achieved through the use of Kronecker delta $(\delta)$ function. Writing the equation of motion of the vibrating cantilever with respect to a

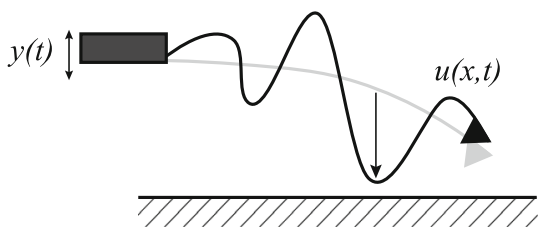

(b)

Fig. 1 Schematic of the AFM cantilever in a static deflection configuration and $\mathbf{b}$ configuration at which AFM cantilever exhibits oscillations around the nonlinear static equilibrium. The sample is at $\bar{\eta}_{1}=1$ 
non-inertial frame of reference leads to the following governing equation,

$$
\begin{aligned}
& \rho A \ddot{u}(x, t)+E I\left(u^{\prime \prime \prime \prime}(x, t)+w^{* \prime \prime \prime \prime}(x)\right) \\
& \quad=F_{i}(Z-w(L, t)) \delta(x-L)+\rho A \Omega^{2} Y \sin (\Omega t),
\end{aligned}
$$

where

$$
\begin{aligned}
F_{i}(Z & -w(L, t)) \\
= & -\frac{A_{1} R}{180\left(Z-w^{*}(L)-u(L, t)-Y \sin (\Omega T)^{8}\right.} \\
& +\frac{A_{2} R}{6\left(Z-w^{*}(L)-u(L, t)-Y \sin (\Omega T)^{2}\right.} .
\end{aligned}
$$

Equation (2) is a non-autonomous and nonlinear equation. The equation is discretized by projecting the dynamics onto the system's linear modes of vibration. The natural modes and frequencies are obtained using the Galerkin approach $[12,23]$. The frequency range of the analyses in this paper spans around the neighbourhood of the fundamental resonance, where the contribution of higher modes is substantially negligible. Based on this assumption, a single-degree-of-freedom model is used. Assuming $u(x, t)=\phi_{1}(x) q_{1}(t)$ (where $\phi_{1}$ is the first approximate eigenfunction around the static deflected configuration) and using the Galerkin approach, the following nonlinear equation can be derived [12]:

$$
\begin{aligned}
\ddot{\bar{\eta}}_{1}= & -d_{1} \dot{\bar{\eta}}_{1}-\bar{\eta}_{1}+B_{1}+\frac{C_{11}}{\left(1-\bar{\eta}_{1}-\bar{y} \sin (\bar{\Omega} t)\right)^{8}} \\
& +\frac{C_{12}}{\left(1-\bar{\eta}_{1}-\bar{y} \sin (\bar{\Omega} t)\right)^{2}}+\bar{y} \bar{\Omega}^{2} E_{1} \sin (\bar{\Omega} t) .
\end{aligned}
$$

The dimensionless variables and the corresponding coefficients are described in "Appendix". The microcantilever tip deflection towards the sample is denoted by $\bar{\eta}_{1}$. In addition, the equation is made dimensionless with respect to equilibrium gap width $\left(\eta^{*}\right)$ and the fundamental frequency of the free microcantilever $\left(\omega_{1}\right)$ in the absence of tip-sample interaction forces. The amplitude of the dither piezoelectric actuator is denoted by $\bar{y}$. The dotted quantities represent derivatives with respect to rescaled time $\tau\left(\tau=\omega_{1} t\right)$. Finally, the modal damping $d_{1}$ is explicitly introduced in Eq. (4) and is related to the quality factor $Q$ of the cantilever by the relation $Q=1 / d_{1}$.

\section{Numerical analysis}

In order to investigate the dynamical behaviour of the TM-AFM, the simulations of the model given by Eq. (4)
Table 1 Properties and dimensions of the cantilever [12]

\begin{tabular}{ll}
\hline Description & Dimensions \\
\hline Length $(L)$ & $449 \mu \mathrm{m}$ \\
Width $(b)$ & $46 \mu \mathrm{m}$ \\
Thickness $(h)$ & $1.7 \mu \mathrm{m}$ \\
Tip radius $(R)$ & $150 \mathrm{~nm}$ \\
Density $(\rho)$ & $2330 \mathrm{~kg} \mathrm{~m}^{-3}$ \\
Static stiffness $(k)$ & $0.11 \mathrm{~N} \mathrm{~m}^{-1}$ \\
Elastic modulus $(E)$ & $176 \mathrm{GPa}$ \\
Fundamental resonance frequency $\left(f_{1}\right)$ & $11.804 \mathrm{kHz}$ \\
Quality factor $(Q)$ & 100 \\
Hamaker constant repulsive $\left(A_{1}\right)$ & $1.35961 \times 10^{-70} \mathrm{~J} \mathrm{~m}^{6}$ \\
Hamaker constant attractive $\left(A_{2}\right)$ & $1.8651 \times 10^{-19} \mathrm{~J}^{6}$ \\
\hline
\end{tabular}

are performed in this section. The entire analysis is carried out for the interaction of a soft monocrystalline silicon microcantilever with the (111) face of flat silicon sample. The cantilever and interaction properties are listed in Table 1. Furthermore, the analysis is performed for tip-sample gap $\left(\eta^{*}\right)$ in the bi-stable region of nonlinear elastostatic equilibrium curve. Thus, a value of $\eta^{*}=6.542 \mathrm{~nm}^{1}$ is chosen for the rest of the analysis in this article. However, a similar analysis can be carried out for any other tip-sample gap values.

Numerical simulations are performed by using a pseudo-arc-length continuation technique [24]. We also make use of basins of attraction (phase space) in order to illustrate the presence of various attractors (steady-state solutions). A basin of attraction is a set of possible initial conditions about an equilibrium point in phase space that assures a specific response from the cantilever. In other words, any chosen initial condition within the phase space will be 'attracted' to a particular steady-state motion of the cantilever. Unless specified, the basins are evaluated in a phase-space grid of $\bar{\eta}_{1}=[-0.9,0.9]$ and $\dot{\bar{\eta}}_{1}=[-0.9,0.9]$ as it contains all the main attractors involved in the system potential well.

\footnotetext{
1 This value allows for comparison of results with the reference paper of Ruetzel et al. [12]. However, qualitatively the same results are obtained for $\eta^{*}=6.5 \mathrm{~nm}$.
} 


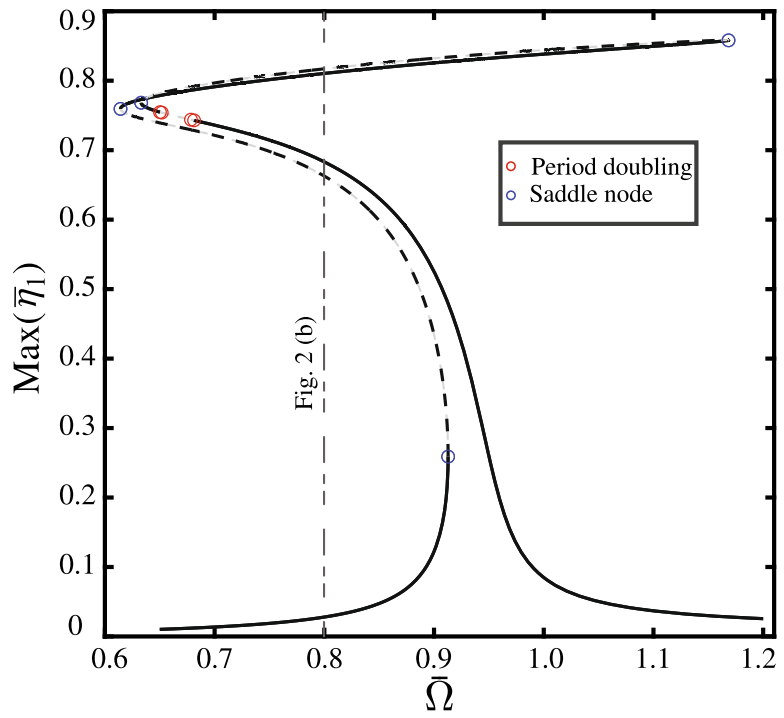

(a)

Fig. 2 Frequency response curve and basin portrait of the system for fixed parameters $\bar{y}=0.006$ and $R=150 \mathrm{~nm}$. The parameters are obtained from the monostable region of nonlinear elastostatic equilibrium curve. a Frequency response shows softening and hardening behaviour corresponding to the attractive and repulsive tip-sample forces. Continuous and dotted lines indicate stable and unstable branches of the solution. Red and

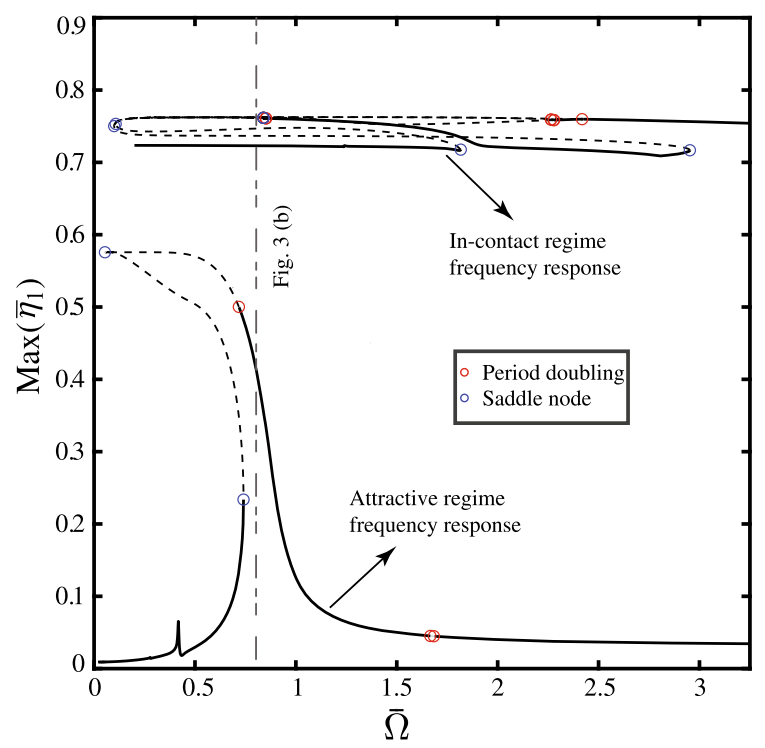

(a)

Fig. 3 Frequency response curve and basin portrait of the system for fixed parameter $\bar{y}=0.020$ and $R=150 \mathrm{~nm}$. The parameters are obtained from the bi-stable region of nonlinear elastostatic equilibrium curve. a Frequency response of the system showing both attractive (lower curve) and in-contact (upper curve) main solutions. Continuous and dotted lines indicate stable and

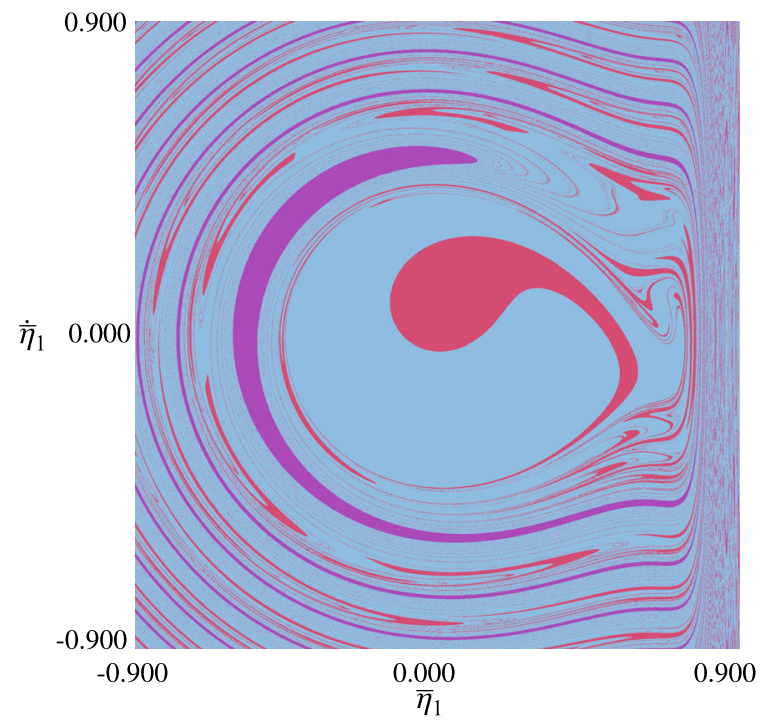

(b)

blue circles indicate period-doubling and saddle-node bifurcation points, respectively. The natural frequency of the system is $\bar{\Omega}_{0}=0.94$. b Basins of attraction taken at section $\bar{\Omega}=0.8$. The positive displacement implies movement of the tip closer to the sample. The details on basin colour and the corresponding attractor/solution description are given in Table 4. (Color figure online)

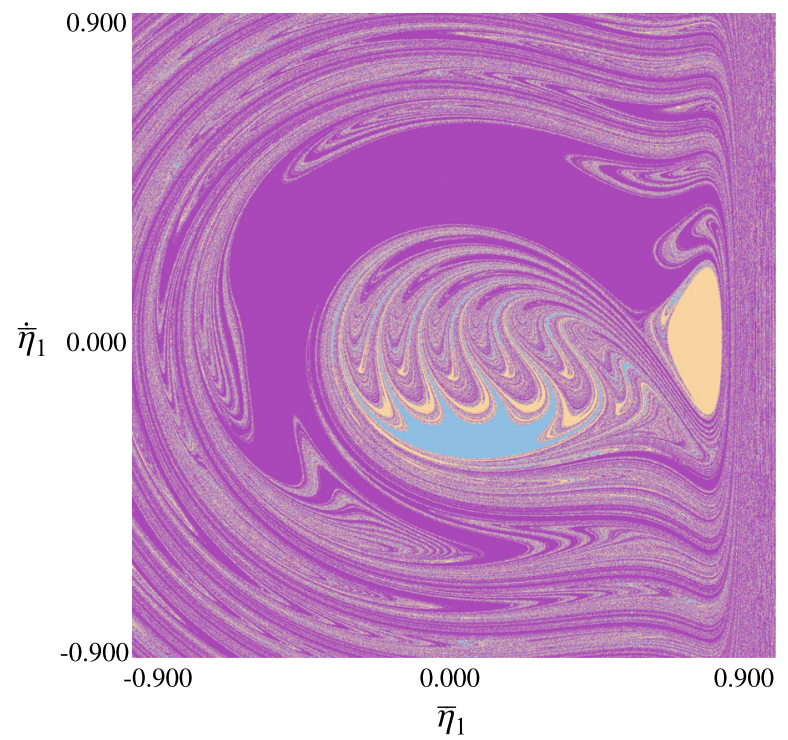

(b)

unstable branches of the solution. Red and blue circles indicate period-doubling and saddle-node bifurcation points, respectively. $\mathbf{b}$ Basins of attraction of the system are obtained at section $\bar{\Omega}=0.8$. The details on basin colour and the corresponding attractor/solution description are given in Table 4. (Color figure online) 


\subsection{Frequency response and bifurcation scenarios}

The interaction between tip and sample gives rise to different nonlinear frequency responses depending on the tip-sample separation distance. The response displayed in Fig. 2a shows an initial softening behaviour when the tip is far away from the sample. This region is dominated by attractive Van der Waals forces. However, when the tip-sample separation reaches the order of the interatomic distance, the response exhibits hardening behaviour and this region is dominated by repulsive forces (see Fig. 2a). Figure $2 b$ shows the corresponding basin portrait associated with the frequency response at $\bar{\Omega}=0.8$. The blue and crimson basins together form the attractive region, while the purple basin belongs to the repulsive region.

The nonlinear frequency response shown in Fig. 2a is well known and studied extensively by many authors $[10,12,25]$. However, there exists another overlooked steady-state response when the cantilever escapes the local potential well and gets trapped by a subsequent attractor very close to the sample. Figure 3 a shows the frequency response for the model given by Eq. (4), and

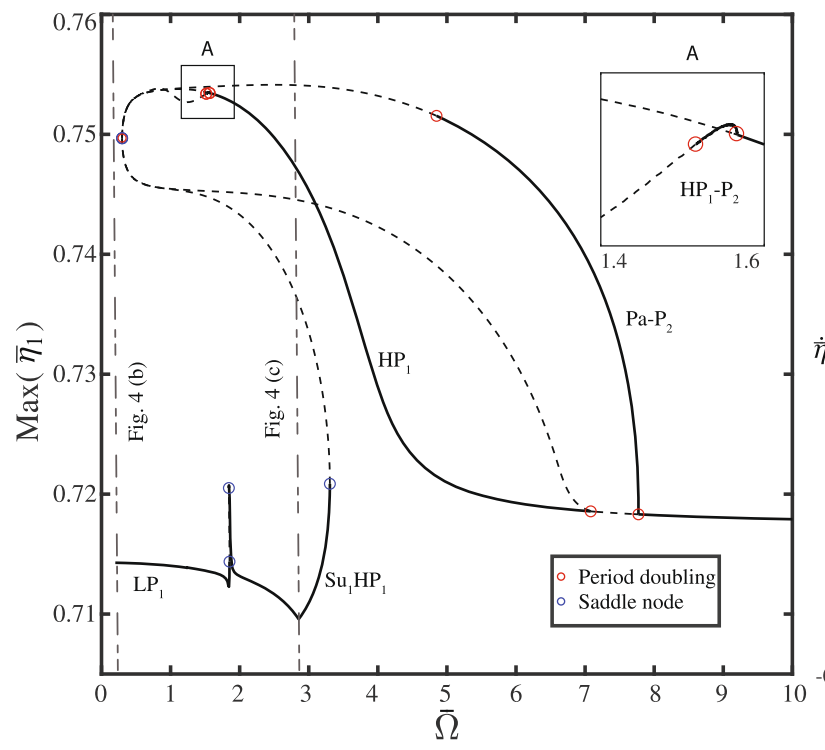

(a) $\bar{y}=0.005$

Fig. 4 Frequency response curve and basins of attraction for the in-contact attractor. a Frequency response of in-contact attractor for fixed parameter $\bar{y}=0.005$ and $R=150 \mathrm{~nm}$. Continuous and dotted lines indicate stable and unstable branches of solution. Red and blue circles indicate period-doubling and saddle-node bifur-
Fig. $3 b$ shows the position of the in-contact attractor in the basin portrait. Note that Fig. 3a is made up of two different solutions belonging to the attractive (lower frequency response curve) and the in-contact attractor (upper frequency response curve). The two solutions can be obtained by using different initial conditions in the numerical integration. In the next section, the detailed analysis of the in-contact attractor's frequency response and corresponding bifurcation scenarios is presented parametrically.

\subsection{Frequency response curves}

Local dynamic analysis is performed using frequency response curves together with the bifurcation charts. The analysis offers a complete overview into the bifurcations and escape scenarios of the system. Furthermore, the unstable solution branches shown in the frequency response curves are not discussed in detail but are reported for the sake of completeness.

Figures 4 and 5 show the evolution of frequency response of the in-contact attractor as a function of the excitation amplitude, $\bar{y}=0.005$ and 0.020 , respec-

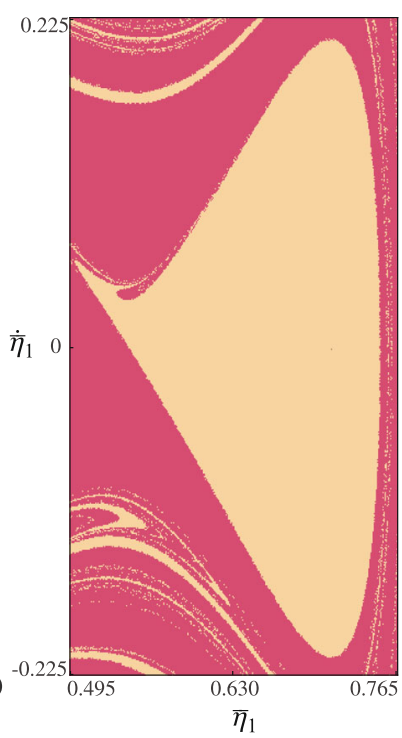

(b) $\bar{\Omega}=0.5$

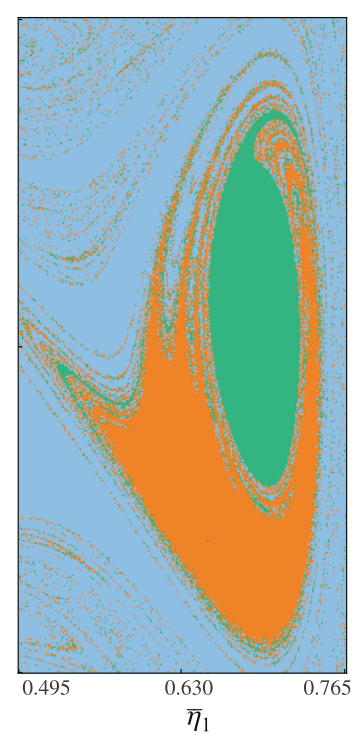

(c) $\bar{\Omega}=2.96$ cation points, respectively. b, $\mathbf{c}$ Basins of attraction of in-contact attractor obtained at sections, $\bar{\Omega}=0.5$ and $\bar{\Omega}=2.96$, respectively, in the frequency response curve. The details on basin colour and the corresponding attractor/solution description are given in Table 4. (Color figure online) 


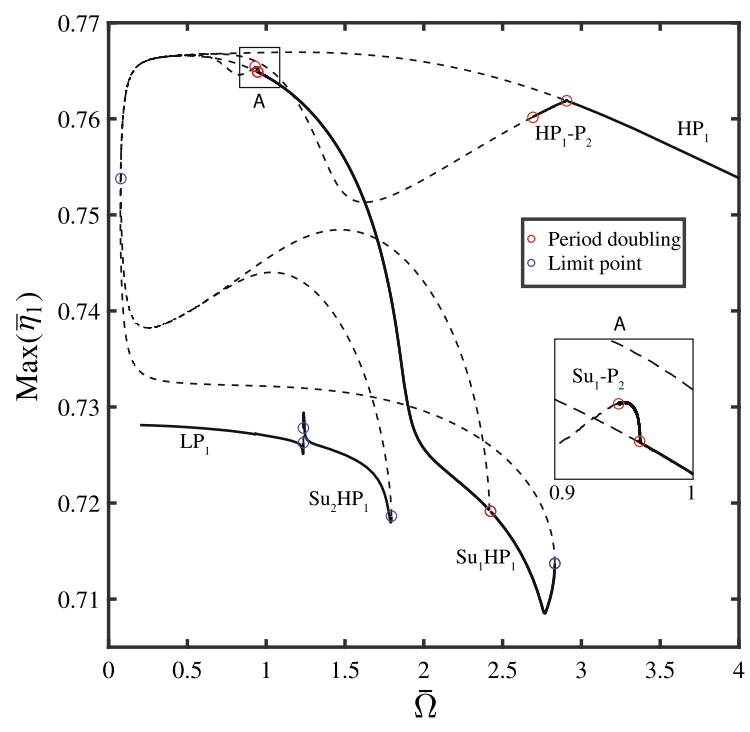

Fig. 5 Frequency response curve of the in-contact attractor for fixed parameter values $\bar{y}=0.020$ and $R=150 \mathrm{~nm}$. Continuous and dotted lines indicate stable and unstable branches of the solution, respectively. Red and blue circles indicate period-doubling and saddle-node bifurcation points, respectively. (Color figure online)

tively. In both figures, red and blue circles indicate period-doubling and saddle-node bifurcation points, respectively. Although the dimensionless natural frequency of the system oscillating in attractive regime is found to be $\bar{\Omega}_{0}=0.83\left(\bar{\Omega}_{0}\right.$ is the natural frequency affected by the system potential well), it can be observed in Fig. 4 that the first natural frequency of the system oscillating in the in-contact regime is $\bar{\Omega}_{0}=3.72$ with corresponding parametric resonance at $2 \bar{\Omega}_{0}=7.44$. This shift in resonance frequency is due to the presence of strong repulsive forces which act as a hard spring connecting the cantilever to the sample. This can be visualized as a change in the boundary conditions of the cantilever similar to that of a clamped-clamped beam. Interestingly, the system also exhibits softening nonlinearity in spite of the presence of repulsive forces. This is due to the fact that the cantilever is oscillating in the potential well with a duration of oscillation longer in the attractive regime. In addition, multi-stability can be observed with different solutions overlapping in several discrete ranges of frequencies (see Fig. 4a). At lower values of forcing frequency $\bar{\Omega} \leq 1.85$, the system has only one non-resonant low-amplitude solution $\left(\mathrm{LP}_{1}\right)$. The corresponding basin associated with $\mathrm{LP}_{1}$ solution at $\bar{\Omega}=0.5$ is shown in light brown colour in Fig. 4 b.
The figure illustrates the low-amplitude attractor being the dominant solution in the in-contact regime at lowfrequency values. The $\mathrm{LP}_{1}$ solution eventually gives rise to a superharmonic branch $\left(\mathrm{Su}_{1} \mathrm{HP}_{1}\right)$ at $\bar{\Omega}=1.85$ via saddle-node bifurcation, and later a resonant highamplitude solution $\left(\mathrm{HP}_{1}\right)$ at $\bar{\Omega}=3$.3. Figure 4 c reports the orange basin belonging to $\mathrm{HP}_{1}$ solution arising from the boundaries of $\mathrm{Su}_{1} \mathrm{HP}_{1}$ solution (green basin) in the in-contact regime.

Furthermore, the $\mathrm{HP}_{1}$ branch in Fig. 4a destabilizes with the inception of a pair of period-2 branches via flip/period-doubling bifurcations. One of the perioddoubling bifurcation occurs close to the low-frequency saddle-node bifurcation at $\bar{\Omega}=0.3$, while the other period-doubling bifurcations occur at $\bar{\Omega}=1.56$ (zoomed part of Fig. 4a). This behaviour is similar to the nonlinear cantilever response seen in attractive regime as illustrated earlier in Fig. 3a. Moreover, in Fig. $4 \mathrm{a}$ the period-2 branch continuation shows stable motion over a short frequency range before undergoing further period-doubling bifurcation cascade. The subharmonic response associated with the period-doubling bifurcation can be observed around the principal parametric resonance frequency of $2 \bar{\Omega}_{0}=7.44$. The stable large amplitude period-2 solution (referred to as $\mathrm{Pa}$ $\mathrm{P}_{2}$ in Fig. 4a), arising from one of the period-doubling points, is found to be stable over a wide range of excitation frequency $(\bar{\Omega} \in[4.8,7.7])$. In addition to the above analysis, referring to Fig. 5, at larger excitation amplitudes $(\bar{y} \geq 0.0118)$, the softening behaviour in the nonlinear response of the system increases along with a larger field of existence of the superharmonic response $\left(\mathrm{Su}_{1} \mathrm{HP}_{1}\right)$. Furthermore, a second superharmonic branch $\left(\mathrm{Su}_{2} \mathrm{HP}_{1}\right)$ bifurcates through a saddlenode at $\bar{\Omega}=1.79$. Here, four forced period- 1 solutions coexist out of which only two are stable $\left(\mathrm{Su}_{2} \mathrm{HP}_{1}\right.$ and $\mathrm{Su}_{1} \mathrm{HP}_{1}$ ) and two are unstable. The superharmonic branch for larger excitations eventually joins the main branch of the in-contact response via the saddle-node at $\bar{\Omega}=2.83$. In addition, the $\mathrm{Su}_{1} \mathrm{HP}_{1}$ branch is destabilized over narrow frequency ranges, $\bar{\Omega} \in[0.92,0.95]$ and $\bar{\Omega} \in[2.41,2.42]$, by the occurrence of perioddoubling bifurcations.

It is observed in Fig. 5 that the low-frequency perioddoubling point $(\bar{\Omega}=0.94$ ) has a stable period-2 solution over a short frequency range (see zoomed part of the figure) and the high-frequency period-doubling $(\bar{\Omega}=2.42)$ presents mostly an unstable bifurcated period-2 solution except for few initial continuation 


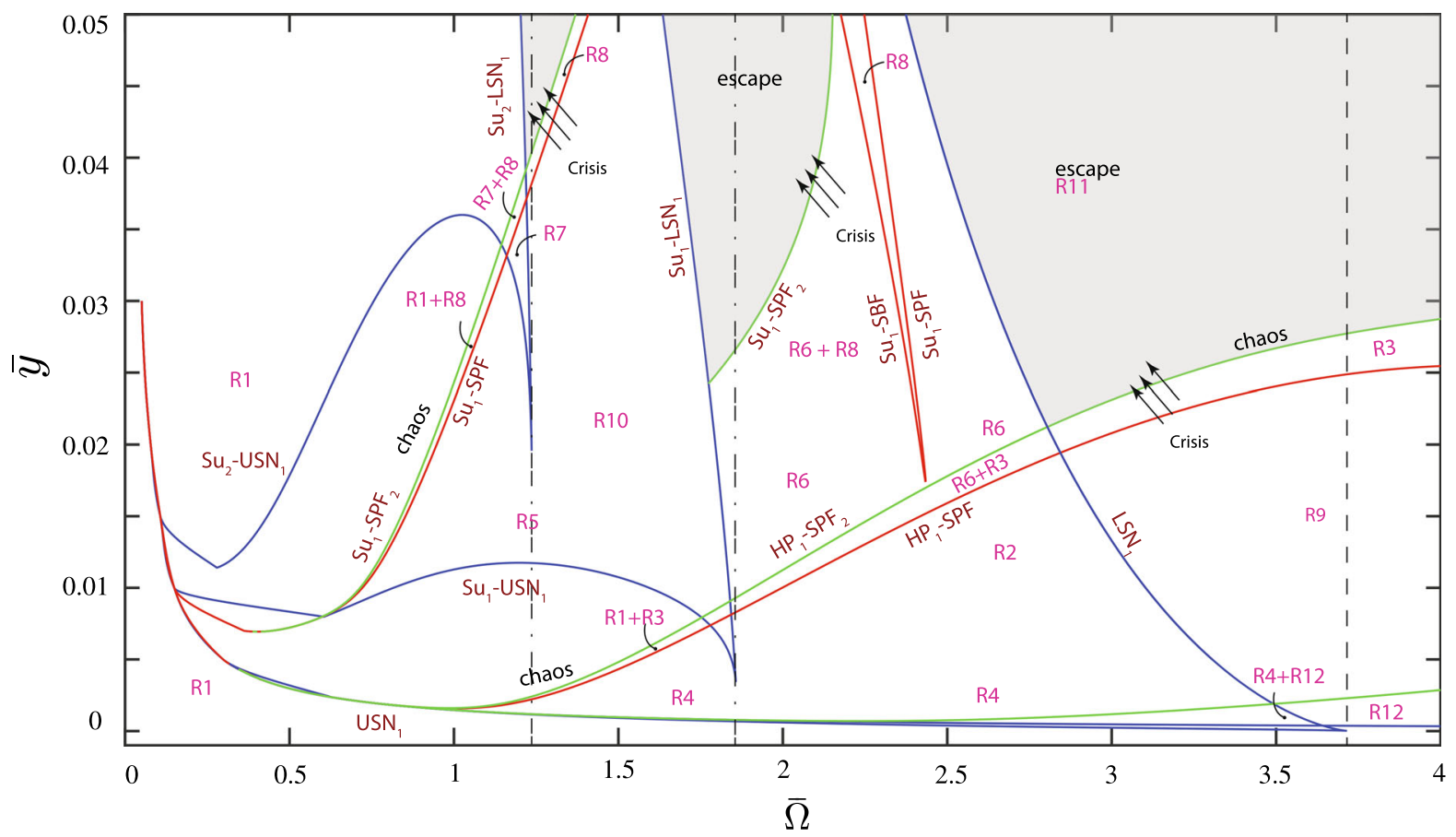

Fig. 6 In-contact attractor bifurcation and response chart focusing on the region below the primary resonance frequency $\left(\bar{\Omega}_{0}=\right.$ 3.721 indicated by the vertical dashed line). The first and second superharmonic frequencies are indicated at $\bar{\Omega}=1.851$ and $\bar{\Omega}=1.24$ by dashed-dotted lines. Blue lines are the saddlenode bifurcation loci on period-1 solution branches, red lines are the period-doubling/flip bifurcation loci on period-1 solution

points that are stable. These few stable points are not shown in the frequency response curves but are described in the bifurcation maps. In an analogous way, a period-doubling bifurcation at $\bar{\Omega}=2.90$ is observed on the $\mathrm{HP}_{1}$ branch having a stable period2 solution limited in frequency range $\bar{\Omega} \in[2.7,2.9]$. Moreover, period-doubling cascades are present in both stable period-2 solutions arising from $\mathrm{Su}_{1} \mathrm{HP}_{1}$ and $\mathrm{HP}_{1}$ branches. This period-doubling cascade can lead to chaos in a similar fashion as encountered in standard TM-AFM systems [26].

\subsection{Bifurcation chart, response scenarios and escape threshold}

Figures 6 and 7 provide an overview of the various bifurcation scenarios and escape thresholds occurring for a wide range of excitation amplitudes and frequencies. In these figures, local bifurcation envelope (loci) is constructed by following the variation of branches, and green lines are the period-doubling/flip bifurcation loci on period- 2 solution branches. The details on individual bifurcation envelope description and the corresponding solution regions are summarized in Tables 2 and 3, respectively. Refer to "Appendix" for detailed instruction on how to read the bifurcation chart. (Color figure online)

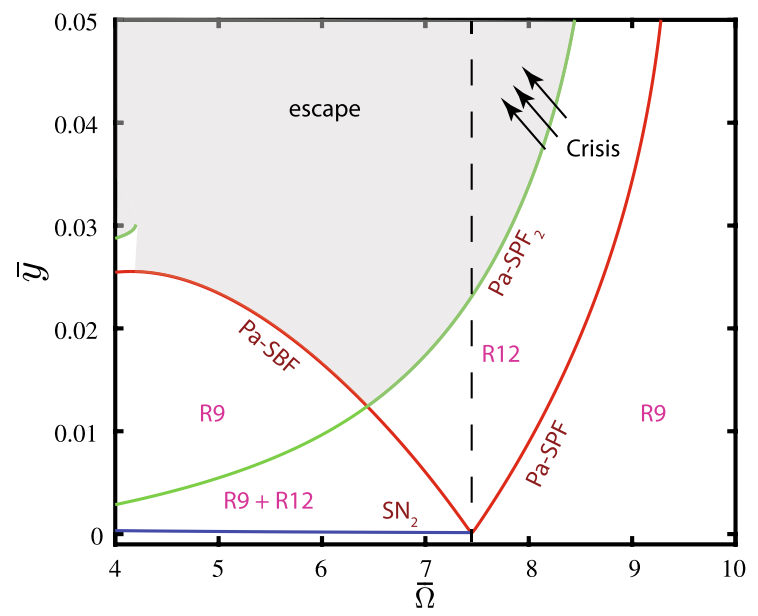

Fig. 7 In-contact attractor bifurcation and response map focusing on the region near the neighbourhood of parametric resonance frequency $\left(2 \bar{\Omega}_{0}=7.44\right.$ indicated by the vertical dashed line). The details on individual bifurcation envelope description and the corresponding solution regions are summarized in Tables 2 and 3 , respectively 
Table 2 Bifurcation envelope data

\begin{tabular}{|c|c|c|}
\hline Envelope name & Envelope colour & Description \\
\hline $\mathrm{USN}_{1} / \mathrm{LSN}_{1}$ & Blue & Upper/lower saddle-node bifurcation loci belonging to the main solution branch \\
\hline $\mathrm{Su}_{1}-\mathrm{USN}_{1} / \mathrm{Su}_{1}-\mathrm{LSN}_{1}$ & Blue & $\begin{array}{l}\text { Upper/lower saddle-node bifurcation loci belonging to the first superharmonic solution } \\
\text { branch }\end{array}$ \\
\hline $\mathrm{Su}_{2}-\mathrm{USN}_{1} / \mathrm{Su}_{2}-\mathrm{LSN}_{1}$ & Blue & $\begin{array}{l}\text { Upper/lower saddle-node bifurcation loci belonging to the second superharmonic } \\
\text { solution branch }\end{array}$ \\
\hline $\mathrm{SN}_{2}$ & Blue & $\begin{array}{l}\text { Saddle-node bifurcation loci belonging to the period- } 2 \text { solution of the principal } \\
\text { parametric resonance }\end{array}$ \\
\hline $\mathrm{Pa}-\mathrm{SBF} / \mathrm{Pa}-\mathrm{SPF}$ & Red & $\begin{array}{l}\text { Subcritical/supercritical flip bifurcation loci emerging from the principal parametric } \\
\text { resonance }\end{array}$ \\
\hline $\mathrm{Su}_{1}-\mathrm{SBF} / \mathrm{Su}_{1}-\mathrm{SPF}$ & Red & $\begin{array}{l}\text { Subcritical/supercritical flip bifurcation loci emerging from the first superharmonic } \\
\text { solution branch }\end{array}$ \\
\hline $\mathrm{Pa}-\mathrm{SPF}_{2}$ & Green & $\begin{array}{l}\text { Supercritical flip bifurcation loci emerging from the period-2 response of the principal } \\
\text { parametric resonance }\end{array}$ \\
\hline $\mathrm{Su}_{1}-\mathrm{SPF}_{2}$ & Green & $\begin{array}{l}\text { Supercritical flip bifurcation loci emerging from the first superharmonic branch with } \\
\text { period- } 2 \text { response }\end{array}$ \\
\hline
\end{tabular}

the bifurcation point (saddle-node or period-doubling) with respect to operating parameters, namely $\bar{\Omega}$ and $\bar{y}$. Furthermore, by assembling all the local bifurcation envelopes together, the global response and behaviour map of the entire system in the excitation amplitude and frequency control space are obtained. The bifurcation map has been obtained numerically for the incontact attractor over a wide range of frequencies which includes the fundamental $\left(\bar{\Omega}_{0}\right)$ and principal parametric resonances $\left(2 \bar{\Omega}_{0}\right)$. Thus, it is convenient to analyse the global dynamics by dividing the bifurcation map into two separate regions: the first region focuses around the fundamental resonance frequency $\bar{\Omega}_{0}=3.72$ illustrated in Fig. 6, whereas the second region analyses the principal parametric resonance frequency $\bar{\Omega}_{0}=7.44$ as shown in Fig. 7. In addition, Table 2 outlines the data concerning the various bifurcation envelopes of Figs. 6 and 7. Furthermore, Table 3 summarizes the various dynamic regions formed by these envelopes and the corresponding solutions involved. From an experimental perspective, the response scenario map provides qualitative information on the form of cantilever response expected for the chosen set of excitation amplitude and excitation frequency.

\subsubsection{Analysis of the bifurcation map around the fundamental resonance}

Around the fundamental resonance frequency of the in-contact attractor, the dynamics is more involved than in the case of the attractive region [19]. The period-doubling/flip bifurcations appear not only on the main branch of the solution ( $\mathrm{HP}_{1}$ branch in Fig. 5), but also on the first superharmonic branch $\left(\mathrm{Su}_{1} \mathrm{HP}_{1}\right.$ branch in Fig. 5). This drastically increases the possibility of global escape through crisis and also chaotic behaviour through period-doubling cascade. In an analogous way, the saddle-node bifurcations that arise from the superharmonic branch increase the complexity of the response.

Figure 6 reports all the possible regions of motions for the cantilever in the range of frequencies surrounding the fundamental resonance. The different regions are named with pink labels and accordingly numbered. For both low-amplitude excitations and frequencies up to $\bar{y}=0.004$ and $\bar{\Omega}<1.85$, there exists only the $\mathrm{LP}_{1}$ motion indicated by region R1 in Fig. 6. Hereafter, with the increase in $\bar{\Omega} \geq 1.85$, the response consists of both the $\mathrm{HP}_{1}$ and superharmonic high-amplitude $\left(\mathrm{Su}_{1} \mathrm{HP}_{1}\right)$ solutions bound by loci $\mathrm{HP}_{1}-\mathrm{SPF}$ as shown in the region R2 of Fig. 6. Moving to even larger values of excitation frequency, the solution $\mathrm{HP}_{1}$ governs the behaviour of the system (R9). In particular, special attention should be given to the regions bounded by SPF (red) and $\mathrm{SPF}_{2}$ (green) loci. These regions exist over a narrow frequency range and invade into other period-1 regions resulting in new multi-stable regions which consist of both period-1 and period-2 solutions. Similarly, in addition to the discussion of aforementioned regions, the presence of other response scenarios and dynamic 
Table 3 Correlation data between the dynamic regions and corresponding solutions

\begin{tabular}{|c|c|c|}
\hline Dynamic region & Solution & Description \\
\hline $\mathrm{R} 1$ & $\mathrm{LP}_{1}$ & Low-amplitude non-resonant period-1 response from the main solution branch \\
\hline $\mathrm{R} 2$ & $\mathrm{Su}_{1} \mathrm{HP}_{1}+\mathrm{HP}_{1}$ & $\begin{array}{l}\text { High-amplitude period-1 solution arising from the first superharmonic branch } \\
\text { and High-amplitude resonant period-1 response arising from main solution } \\
\text { branch }\end{array}$ \\
\hline R3 & $\mathrm{HP}_{1}-\mathrm{P}_{2}$ & Period-2 response arising from the resonant high-amplitude solution \\
\hline R4 & $\mathrm{LP}_{1}+\mathrm{HP}_{1}$ & $\begin{array}{l}\text { Low-amplitude period-1 and High-amplitude period- } 1 \text { solution arising from } \\
\text { the main branch }\end{array}$ \\
\hline R5 & $\mathrm{LP}_{1}+\mathrm{Su}_{1} \mathrm{HP}_{1}$ & $\begin{array}{l}\text { Low-amplitude period-1 response belonging to the main solution branch } \\
\text { together with high-amplitude period-1 solution arising from the first } \\
\text { superharmonic branch }\end{array}$ \\
\hline R6 & $\mathrm{Su}_{1} \mathrm{HP}_{1}$ & High-amplitude period- 1 solution arising from the first superharmonic branch \\
\hline R7 & $\mathrm{LP}_{1}+\mathrm{Su}_{1} \mathrm{HP}_{1}+\mathrm{Su}_{2} \mathrm{HP}_{1}$ & $\begin{array}{l}\text { Low-amplitude period-1 response together with high-amplitude period-1 } \\
\text { solution arising from the first and second superharmonic branches, } \\
\text { respectively }\end{array}$ \\
\hline $\mathrm{R} 8$ & $\mathrm{Su}_{1}-\mathrm{P}_{2}$ & Period-2 solution arising from the first superharmonic branch \\
\hline R9 & $\mathrm{HP}_{1}$ & High-amplitude resonant period-1 response arising from main solution branch \\
\hline $\mathrm{R} 10$ & $\mathrm{Su}_{1} \mathrm{HP}_{1}+\mathrm{Su}_{2} \mathrm{HP}_{1}$ & $\begin{array}{l}\text { High-amplitude period-1 solution arising from the first and second } \\
\text { superharmonic branches, respectively }\end{array}$ \\
\hline R11 & Escape region & Period-doubling cascades and existence of strange attractors \\
\hline R12 & $\mathrm{Pa}-\mathrm{P}_{2}$ & Period- 2 response from the principal parametric resonance solution \\
\hline
\end{tabular}

Table 4 Correlation data between the basin colours and corresponding attractors

\begin{tabular}{|c|c|c|c|}
\hline Basin color & $\begin{array}{l}\text { Attractor/solution } \\
\text { branch }\end{array}$ & Basin color & $\begin{array}{l}\text { Attractor/solution } \\
\text { branch }\end{array}$ \\
\hline & A-LP 1 & & $\mathrm{~A}-\mathrm{HP}_{1}$ \\
\hline & Repulsive solution & & $\mathrm{LP}_{1}$ \\
\hline & $\mathrm{Su}_{1}-\mathrm{HP}_{1}$ & & $\mathrm{HP}_{1}$ \\
\hline & A-Pa- $\mathrm{P}_{2}$ and $\mathrm{Pa}-\mathrm{P}_{2}$ & & \\
\hline
\end{tabular}

regions data is tabulated in Table 3. Moreover, in contrast to the various stable responses seen in Fig. 6, the bifurcation analysis of in-contact attractor shows the presence of multiple strange attractors leading to crisis scenarios and global escape. The operating parameters leading to escape are depicted by grey regions (R11) in Fig. 6. The crisis scenario also highlights appearance of several rare attractors (period-5 and above) along with period-doubling cascades, which lead the solution to escape from the local potential well.
The detailed instructions on reading the bifurcation chart together with an example are provided in "Appendix B".

\subsubsection{Analysis of the bifurcation map around the principal parametric resonance}

Figure 7 shows that, below the parametric resonance frequency, $\bar{\Omega}<7.44$, only $\mathrm{HP}_{1}$ solution exists (R9) 
until the $\mathrm{Pa}-\mathrm{P}_{2}$ solution from the parametric resonance $\left(2 \bar{\Omega}_{0}=7.44\right)$ overlaps with the region $\mathrm{R} 9$ resulting in two coexisting period-1 $\left(\mathrm{HP}_{1}\right)$ and period-2 $\left(\mathrm{Pa}-\mathrm{P}_{2}\right)$ responses as shown in region $\mathrm{R} 9+\mathrm{R} 12$. In between the classic parametric instability tongue (V-shaped region near $\bar{\Omega}=7.44)$ formed by the parametric subcritical (Pa-SBF) and supercritical (Pa-SPF) bifurcations, the $\mathrm{HP}_{1}$ solution becomes unstable and there exists only period-2 (Pa-P2) solution (R12). As expected in any of the archetypal parametric oscillators, the system requires a minimum excitation threshold to achieve parametric resonance. This is indicated by the lifting of the instability tongue ( $\mathrm{V}$-shaped region) along the $\bar{y}$ axis and in our system the critical threshold is at $\bar{y}=0.00014$. Finally, for excitation frequency $\bar{\Omega}>7.44$, only $\mathrm{HP}_{1}$ solution is present as shown in region $\mathrm{R} 9$.

\section{Dynamical integrity and robustness of attractors}

In the previous section, we provided insight into diverse solutions and bifurcation scenarios including escape thresholds. However, the analysis did not furnish details on the various instability paths and eventual escape of the steady-state solutions. The information on the instability path (escape from local potential well, cross-well chaos) taken by the cantilever response is of utmost importance in practical applications of AFM. This helps to disentangle image artefacts from factual data.

From an experimental perspective, if the system perturbations can be quantized, then basins of attraction provide insight into the evolution of various steadystate responses (system attractors) and instabilities (erosion profiles) occurring in the system. Furthermore, measures of the basin portraits, the dynamical integrity of the system, are able to quantify the robustness of different attractors. This can be realized through various scalar integrity measures [27]. These integrity measures provide information on the strength of such quantized perturbations required to destabilize the corresponding system response. Therefore, basin portraits together with integrity measures provide a means to track the basin erosion process with respect to changes in operating parameters. Hence, in order to advance the dynamical analysis of the AFM cantilever in the in-contact regime of oscillation, this section focuses on the global topology analysis by means of basins of attraction [28,29].
There have been multiple integrity measures introduced in the literature [30], and this section makes use of two integrity indicators to measure the evolution of phase-space topology, namely local integrity measures (LIM) [27] and integrity factor [31] (IF). The LIM is defined as the normalized radius of the largest hypersphere (circle in 2D), centred on the safe attractor and entirely belonging to the safe basin. It is used to analyse the robustness of the attractor of interest against perturbations. On the other hand, the IF is defined as the normalized radius of the largest circle entirely belonging to the compact part of safe basin. The IF is suitable to study the dynamical integrity of the attractors subject to perturbation around its initial equilibrium condition. The reason to choose these measures with respect to others such as global integrity measures (GIM) relies on the fact that IF and LIM can disentangle the fractality of basin since they focus only on its compact part [27]. In our case this is a serious advantage since the homoclinic tangling of the saddle results in fractalization of the low-amplitude attractive regime basin.

\subsection{Basin portraits and evolution as a function of tip radius}

One of the common causes for image artefacts during AFM scanning operation is the degradation of tip radius $R$ due to repetitive impacts with the sample surface [32]. Furthermore, the correct and reliable operation of the AFM is dependent on the status of the probe tip, since it is responsible for resolving the topography of the sample [10]. The change in radius value during the aforementioned scanning operation thus causes a sensible variation in the system response and corresponding global integrity values. Therefore, in this section, we make an effort to elucidate the variation of system integrity via basin portraits by considering the AFM tip radius $(R)$ as the corresponding varying parameter.

Figure 8 outlines the variation of dynamical integrity of system attractors as a function of AFM cantilever tip radius $R$. The numerical simulations are performed for tip radius ranging from 105 to $225 \mathrm{~nm}$ in steps of $3 \mathrm{~nm}$, with excitation frequency $\bar{\Omega}=0.85$ being close to resonance, and excitation amplitude $\bar{y}=0.005$. Figure $8 \mathrm{a}-\mathrm{c}$ reports the integrity variation trend as a function of tip radius, and Fig. 8d-f reveals the snapshot of basin portrait at several crucial radius values, namely $114 \mathrm{~nm}, 117 \mathrm{~nm}$, and $197 \mathrm{~nm}$, respectively. The contin- 


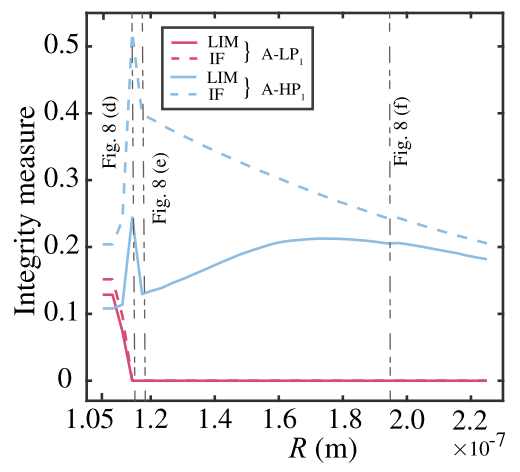

(a) Integrity profiles for attractive regime attractors

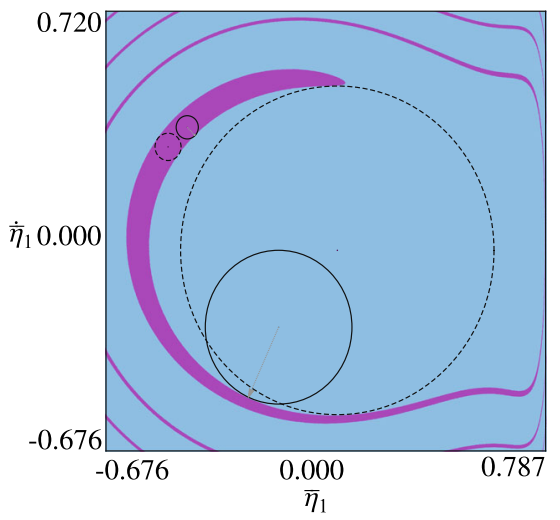

(d) $R=114 \mathrm{~nm}$

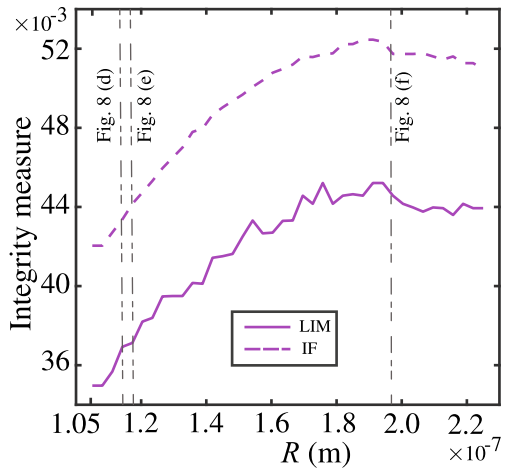

(b) Integrity profiles for repulsive regime attractors

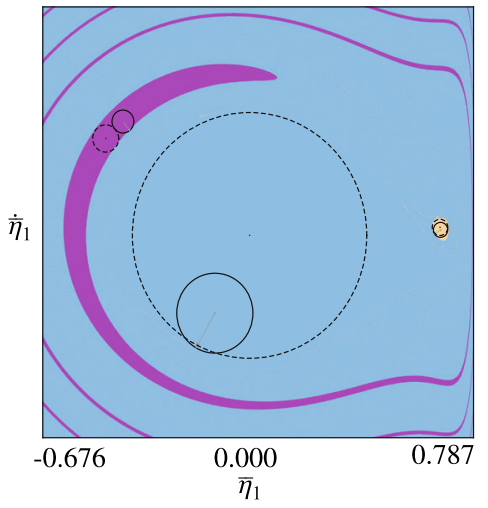

(e) $R=117 \mathrm{~nm}$

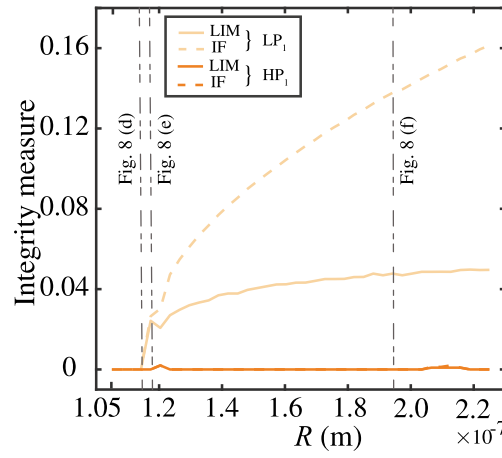

(c) Integrity profiles for in-contact regime attractors

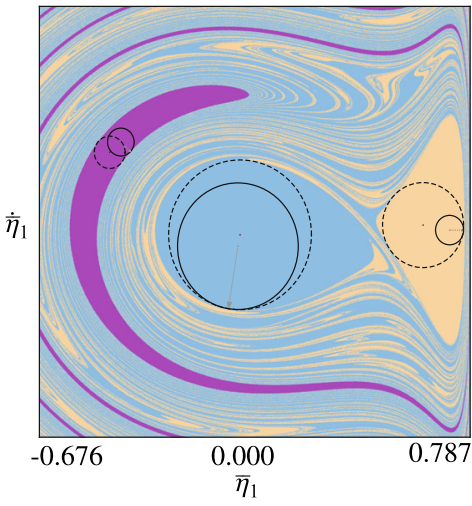

(f) $R=197 \mathrm{~nm}$

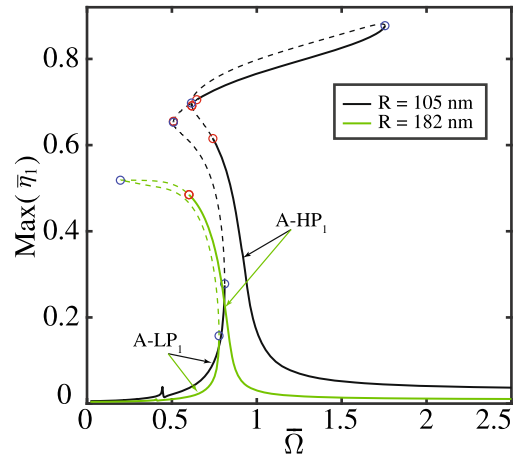

(g) Frequency response curves - attractive regime

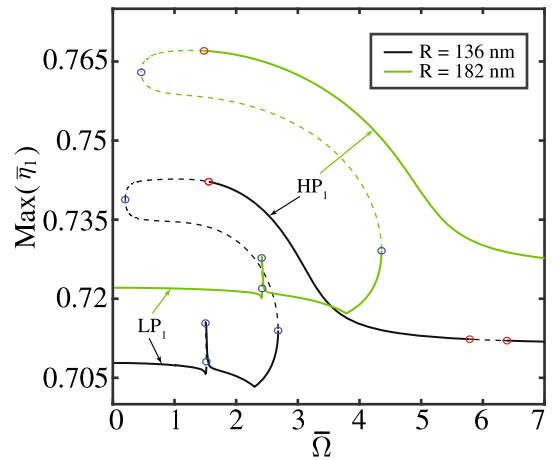

(h) Frequency response curves - in-contact regime
Fig. 8 Variation of integrity measures (LIM, IF), basin portraits and frequency response curves as a function of tip radius $(R)$, for fixed parameter values of $\bar{y}=0.005$ and $\bar{\Omega}=0.85$. a Integrity profiles of the attractive region containing non-resonant low-amplitude (crimson) and resonant high-amplitude solution (blue) attractors. b Integrity profiles of repulsive region attractor. c Integrity profiles of the in-contact region consisting of non-resonant low-amplitude (light brown) and resonant highamplitude solution (orange) attractors. The continuous and dot- ted lines indicate the LIM and IF integrity measures, respectively. d-f show the basin portraits at specific radius values of $114 \mathrm{~nm}$, $117 \mathrm{~nm}$, and $197 \mathrm{~nm}$, respectively. The details on basin colour and the corresponding attractor/solution description are given in Table 4. g Frequency response of microcantilever with radius $R=105 \mathrm{~nm}$ and $R=182 \mathrm{~nm}$ oscillating with initial condition in attractive region. $\mathbf{h}$ Frequency response of microcantilever with radius $R=136 \mathrm{~nm}$ and $R=182 \mathrm{~nm}$ oscillating with initial condition in the in-contact region. (Color figure online) 


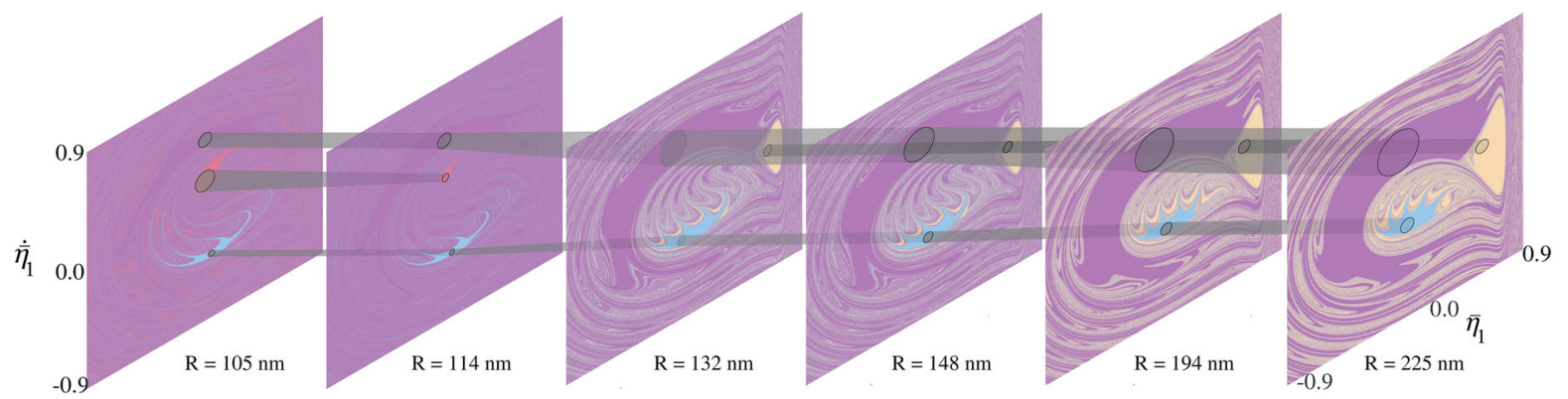

Fig. 9 Phase-space topology evolution with respect to tip radius $(R)$ from 105 to $225 \mathrm{~nm}$, at fixed excitation amplitude and frequency of $\bar{y}=0.020, \bar{\Omega}=0.8$, respectively

uous and dotted lines in Fig. 8a-c belong to LIM and IF measures, respectively.

It is observed in Fig. 8a that in the attractive regime around the radius value $R=114 \mathrm{~nm}$, there is a sharp increase in the high-amplitude resonant solution (shown in Fig. 8g as A-HP ${ }_{1}$ ) and the integrity of lowamplitude non-resonant solution (shown in Fig. $8 \mathrm{~g}$ as $\mathrm{A}-\mathrm{LP}_{1}$ ) rapidly decreases to zero, indicating the complete erosion of its basin from the system. This phenomenon is observed in the basin portrait of Fig. 8d, where the blue basin representing A-HP ${ }_{1}$ solution completely dominates the attractive region. Physically, the disappearance of $\mathrm{A}-\mathrm{LP}_{1}$ solution marks the transition from a bi-stable to a monostable cantilever response in the attractive regime. Interestingly, in Fig. 8a, by considering an even blunter tip, with radius in the range of $R \in[114,117] \mathrm{nm}$, the robustness of $\mathrm{A}-\mathrm{HP}_{1}$ suddenly drops, due to the appearance of a novel competing attractor in the system. The new attractor is indicated by the light brown basin in Fig. 8e. This novel attractor, which is the in-contact attractor, has a smaller growth rate at lower radius values and does not affect the erosion of the A-HP 1 solution rapidly. This is observed in Fig. 8a by a steady increase in the integrity measure of $\mathrm{A}-\mathrm{HP}_{1}$ solution between radius values $R \in[117,185] \mathrm{nm}$. With the further increase in the blunting of the tip, the A-HP 1 basin is eroded along its boundaries smoothly by the in-contact attractor as seen in Fig. 8f. The robustness characteristics shown by LIM and IF measures for attractive regime follow similar trend. However, the IF safe basin measure is larger in magnitude compared to LIM which is due to the smooth erosion of the basin without in-well fractality at low excitation amplitudes $(\bar{y}<0.015)$.
The repulsive attractor, unlike attractive, shows a steady growth in basin size for increasing radius values as shown in Fig. 8b. This trend of increasing basin size is due to the fact that an increase in radius value will increase the area over which repulsive forces are perceived by the system. However, it is worth to observe in Fig. 8d-f that the size of the purple basin (strength of integrity measure) associated with repulsive attractor is very small compared to the attractive and the in-contact regimes. Thus, at low $\bar{y}$, the repulsive attractor although resilient to changes in radius has a smaller influence on the basin erosion process as compared to other two attractors. Moreover, at low radius values, a perturbation inside the attractive regime (blue basin) can lead the system towards the repulsive attractor leading to hardening behaviour as illustrated in Fig. 8g. In this case, for a cantilever oscillating initially in the attractive region, the system frequency response shows both softening due to attractive forces and hardening due to repulsive forces as shown in Fig. $8 \mathrm{~g}$ (black frequency response curve). However, with a further increase in radius values, the attractive and repulsive basins are separated and the system oscillates purely in attractive regime showing only softening nonlinearity (green frequency response curve in Fig. 8g).

The robustness characteristics displayed by the incontact attractor in Fig. 8c are unique, and present features are not observed in the other two attractors. For low radius values, e.g. $R<117 \mathrm{~nm}$, the in-contact attractor does not exist as seen in Fig. 8c and further illustrated by the absence of light brown basin in Fig. 8d. This highlights the existence of a critical radius value for the manifestation of the in-contact attractor. This critical radius value for our system is at $R=117 \mathrm{~nm}$. Around this radius value, there is a 
sudden appearance of the in-contact attractor (shown in Fig. 8e as light brown basin) and it grows steadily with the increase in the value of $R$. Interestingly, the critical radius value remains the same for higher excitation amplitudes and excitation frequencies. Furthermore, from Fig. 8c it is observed that, the growth rate of low-amplitude solution $\left(\mathrm{LP}_{1}\right)$ is not as steep as the attractive and repulsive attractors, but at large radii, the in-contact attractor eventually becomes dominant with no in-well fractality or boundary erosion.

Figure 9 illustrates the evolution of the basin portrait with respect to the tip radius. The LIM integrity measure is utilized to characterize the robustness of the attractors and track the changes in the basin portraits as the tip deteriorates. The basin portraits are analysed for constant parameters $\bar{y}=0.020$ and $\bar{\Omega}=0.8$. The figure reinforces the previous discussion pictorially. It depicts the disappearance of $\mathrm{A}-\mathrm{LP}_{1}$ basin, fractalization of the attractive regime triggering the erosion process and finally the requirement of critical radius for manifestation of the in-contact attractor.

\subsection{Basin erosion as a function of excitation frequency and excitation amplitude}

In Sect. 4.1, the evolution of phase-space topology as a function of radius was showcased. Accordingly, in this section the dynamical integrity analysis aims at quantifying the extent and evolution of the basins along with their erosion process as a function of the excitation amplitude $(\bar{y})$ and excitation frequency $(\bar{\Omega})$. This is established in Fig. 10 for excitation amplitudes $\bar{y}=0.005, \bar{y}=0.010$ and $\bar{y}=0.020$, respectively, whereas Figs. 11 and 12 report the snapshots of basin portraits at crucial excitation frequencies $(\bar{\Omega})$ near the neighbourhood of fundamental and principal parametric resonance frequencies, respectively.

All simulations are performed at a constant radius value of $R=150 \mathrm{~nm}$ and finally, LIM and IF measures are calculated for the aforementioned data. However, we observed that the strength of LIM and IF measure as a function of $\bar{\Omega}$ remain approximately the same. Thus, we can argue that the excitation frequency in the selected interval does not modify the global shape of the portrait and we do not experience a significant subdivision of the basin. Therefore, in order to simplify the analysis, only LIM is used to quantify the robustness of dominant attractors present in the system.

\subsubsection{Analysis of basin erosion profiles around the fundamental resonance frequency}

The fundamental ideology of TM-AFM is based on the near-resonant excitation of the microcantilever. Therefore, it is of significant interest to study the system topology with regard to basin erosion profiles since, these profiles are indicative of solution instabilities. In this respect, the following section utilizes the integrity measures shown in Fig. 10 to discuss the various system attractors in the neighbourhood of their respective resonance frequencies. In addition, to further delineate the behaviours observed in the integrity profiles, the basin portraits are reported in Fig. 11 at specific frequencies.

The integrity profiles of attractive regime attractors are illustrated in Fig. 10a and, similar to Sect. 4.1, the crimson and blue colours belong to the low-amplitude non-resonant solution $\left(\mathrm{A}-\mathrm{LP}_{1}\right)$ and the high-amplitude resonant solution $\left(\mathrm{A}-\mathrm{HP}_{1}\right)$, respectively. Figure $10 \mathrm{a}$ shows that the attractive regime is dominated by A$\mathrm{LP}_{1}$ non-resonant solution at low excitation frequencies $\bar{\Omega}<0.77$. This is illustrated through the basin portrait of Fig. 11a in which the crimson basin corresponding to $\mathrm{A}-\mathrm{LP}_{1}$ is the dominant solution. By further increasing the excitation frequency value above $\bar{\Omega}>0.77$, the A-LP ${ }_{1}$ solution exhibits a sharp decrease in its integrity value (illustrated in Fig. 10a). The sharp decline is attributed to the sudden appearance of the $\mathrm{A}-\mathrm{HP}_{1}$ resonant attractor from inside the local potential well via saddle-node bifurcation. The appearance of saddle-node triggers the erosion process of the A$\mathrm{LP}_{1}$ basin from outside its boundaries. The appearance of $\mathrm{A}-\mathrm{HP}_{1}$ (blue basin) inside the $\mathrm{A}-\mathrm{LP}_{1}$ local potential well (crimson basin) is shown in Fig. 11b. Any further increment in excitation frequency causes the complete erosion of the low-amplitude attractive $\left(\mathrm{A}-\mathrm{LP}_{1}\right)$ solution as seen in Fig. 11c, leaving large amplitude oscillations of $\mathrm{A}-\mathrm{HP}_{1}$ as the dominant solution in the attractive potential well. Hereafter, the $\mathrm{A}-\mathrm{HP}_{1}$ solution remains largely stable as illustrated in Fig. 10a, and its robustness is mainly affected when the excitation frequency reaches the in-contact fundamental resonance or when the system is driven with an excitation amplitude above the parametric threshold of the system. This trend of solution instabilities observed around the fundamental resonance frequency remains unaffected irrespective of excitation amplitudes.

Contrary to the above discussion on attractive regime, where steady-state solutions exist over a 


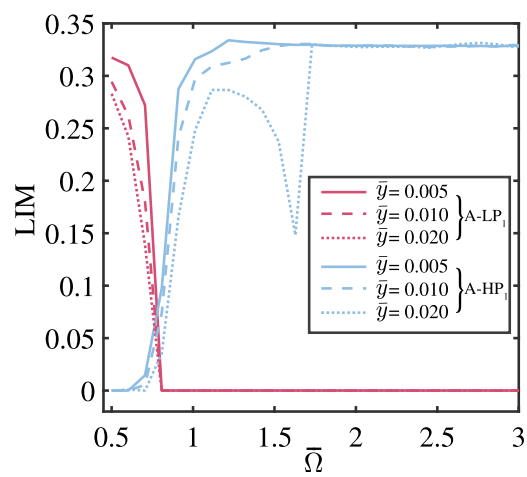

(a) Integrity profiles for attractive regime attractors

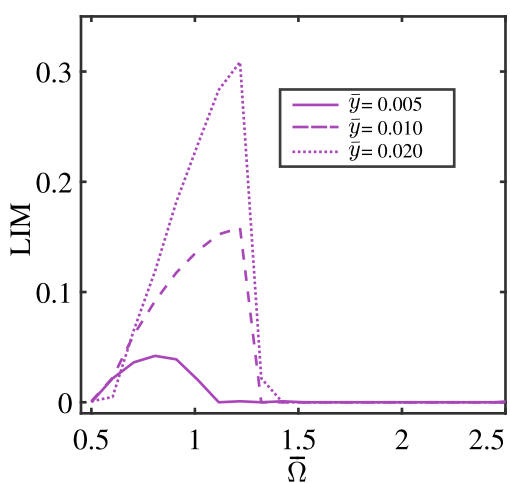

(b) Integrity profiles for repulsive regime attractors

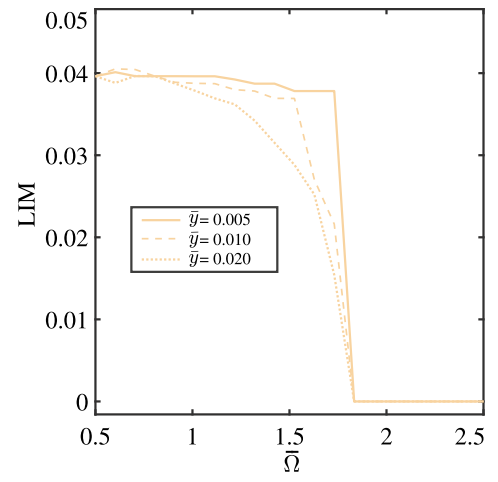

(c) Integrity profiles for $\mathrm{LP}_{1}$ attractor of in-contact regime

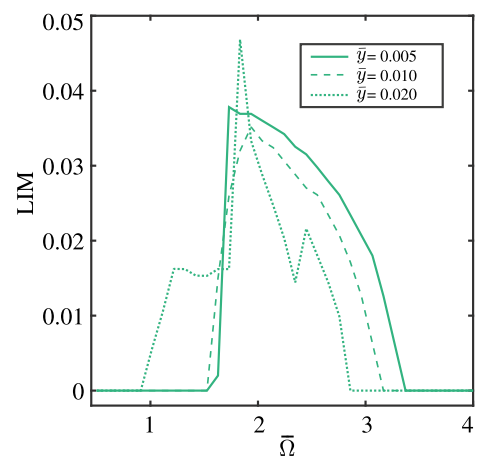

(d) Integrity profiles for $\mathrm{Su}_{1}-\mathrm{HP}_{1}$ attractor of in-contact regime

Fig. 10 Variation of LIM as a function of excitation frequency $(\bar{\Omega})$ for fixed parameter values of $\bar{y}=0.005, \bar{y}=0.010$, $\bar{y}=0.020$ and $R=150 \mathrm{~nm}$. a Integrity profiles for the attractive region consisting of non-resonant low-amplitude (crimson) and resonant high-amplitude branch attractors (blue). b Integrity profiles for repulsive region attractor (purple). $\mathbf{c}$ Integrity profiles

large range of frequencies, the repulsive basin (purple) exists in a narrow frequency range around the fundamental resonance frequency and shows sharp increase/decrease as we move closer/farther away from resonance. This is illustrated in Fig. 10b for $\bar{y}=0.005$, $\bar{y}=0.010$ and $\bar{y}=0.020$, respectively. Moreover, the repulsive basin size is small compared to other two attractors in case of $\bar{y}=0.005$ as shown in Fig. 11c. But it displays a sharp increase in size for higher values of $\bar{y}$ as seen in Fig. 11d for $\bar{y}=0.020$. This is due to the fact that the harder the cantilever is driven, the deeper the oscillations penetrate into the repulsive regime, and the time period of oscillations spent in repulsive regime increases. Therefore, contrary to the

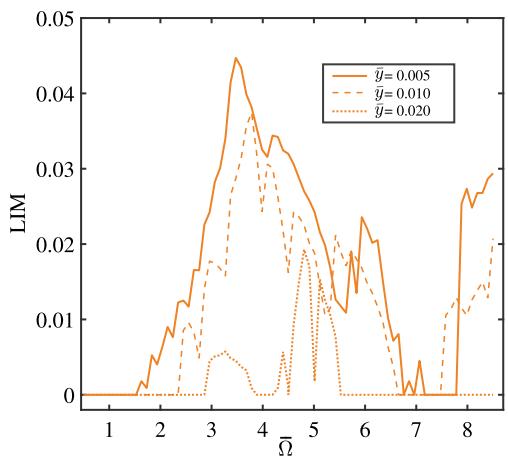

(e) Integrity profiles for $\mathrm{HP}_{1}$ attractor of in-contact regime

for the non-resonant low-amplitude (light brown) attractor in the in-contact region. $\mathbf{d}$ Integrity profiles for the first superharmonic branch attractor in the in-contact region. e Integrity profiles for the resonant high-amplitude branch (orange) attractor in the incontact region. (Color figure online)

observation in Sect. 4.1, the repulsive basin at higher excitation amplitudes significantly constricts the attractive regime basin in the neighbourhood of the fundamental resonance frequency.

On the other hand, the in-contact regime solution displays rich nonlinear behaviour absent in the case of the attractive and repulsive regimes. Figure 10c-e illustrates the integrity profiles of various solution branches namely $\mathrm{LP}_{1}, \mathrm{Su}_{1}-\mathrm{HP}_{1}$, and $\mathrm{HP}_{1}$ that are observed in the in-contact regime. Similar to Sect. 4.1 and summarized in Table 4, the light brown colour corresponds to nonresonant $\mathrm{LP}_{1}$ solution branch, the green colour belongs to $\mathrm{Su}_{1} \mathrm{HP}_{1}$ solution branch and orange colour belongs to $\mathrm{HP}_{1}$ resonant solution branch. The appearance and 


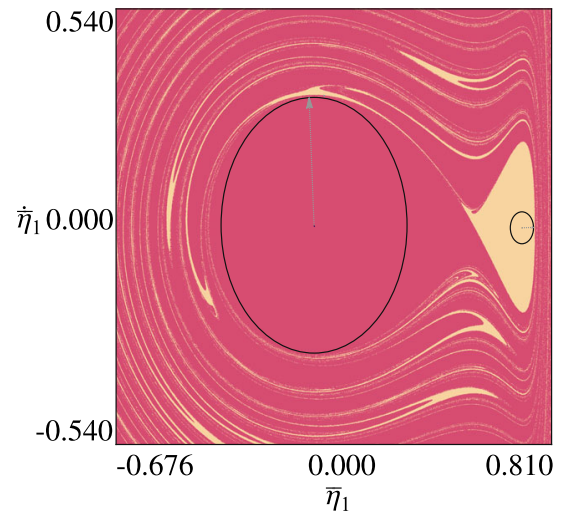

(a) $\bar{y}=0.005, \bar{\Omega}=0.6$

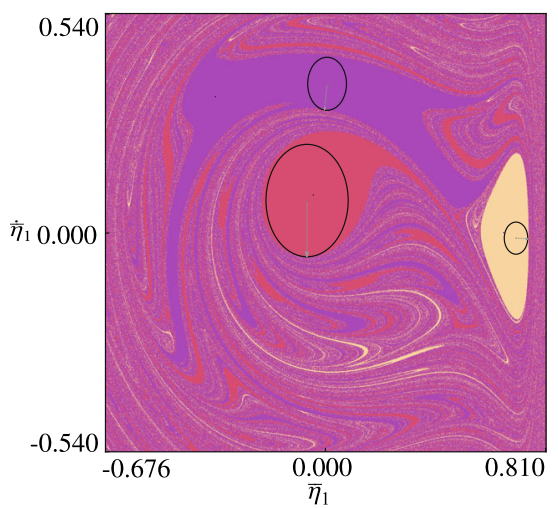

(d) $\bar{y}=0.020, \bar{\Omega}=0.7$

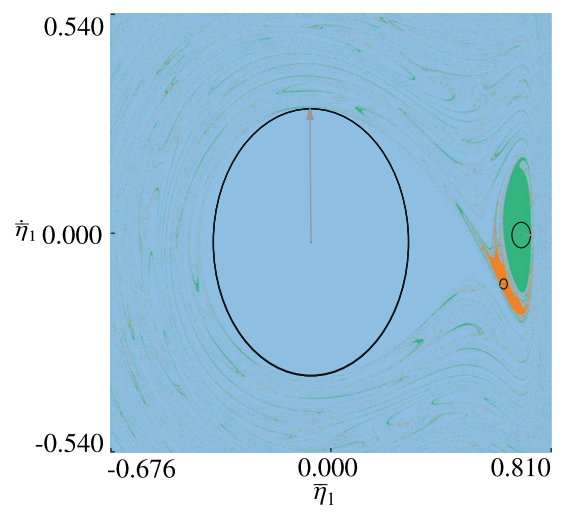

(g) $\bar{y}=0.005, \bar{\Omega}=2.5$

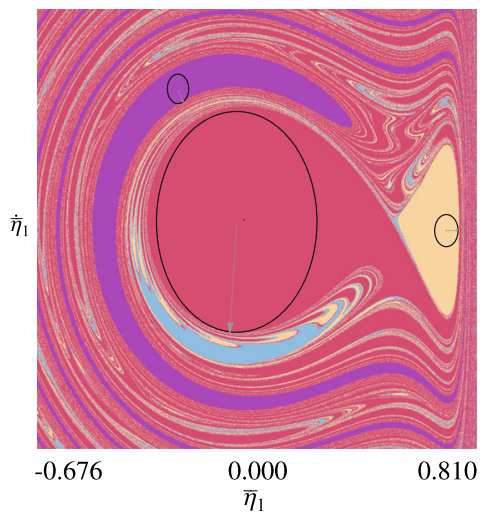

(b) $\bar{y}=0.005, \bar{\Omega}=0.8$

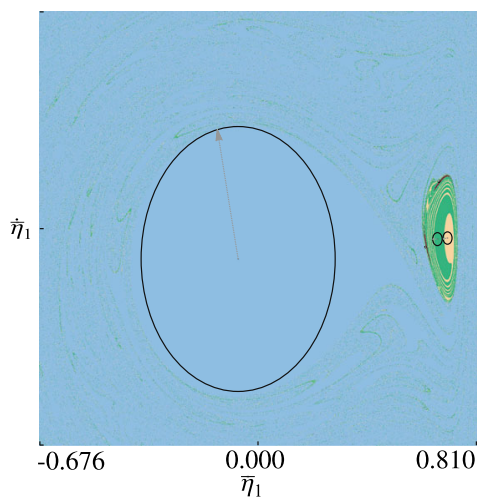

(e) $\bar{y}=0.020, \bar{\Omega}=1.8$

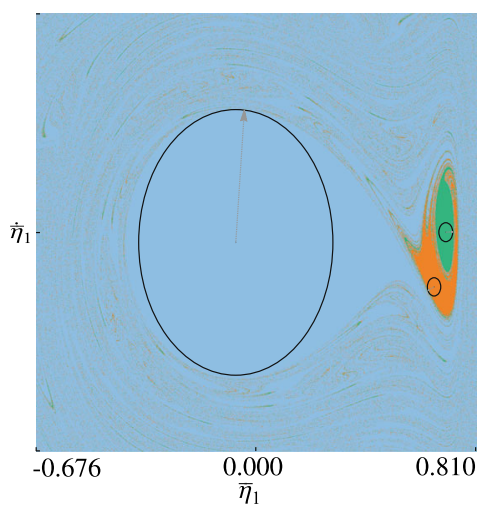

(h) $\bar{y}=0.005, \bar{\Omega}=2.9$

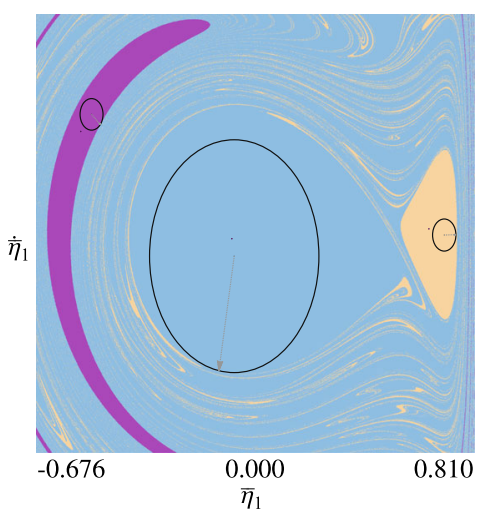

(c) $\bar{y}=0.005, \bar{\Omega}=0.9$

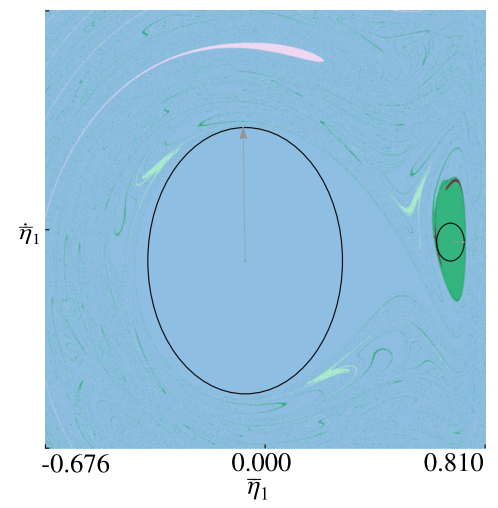

(f) $\bar{y}=0.020, \bar{\Omega}=1.9$

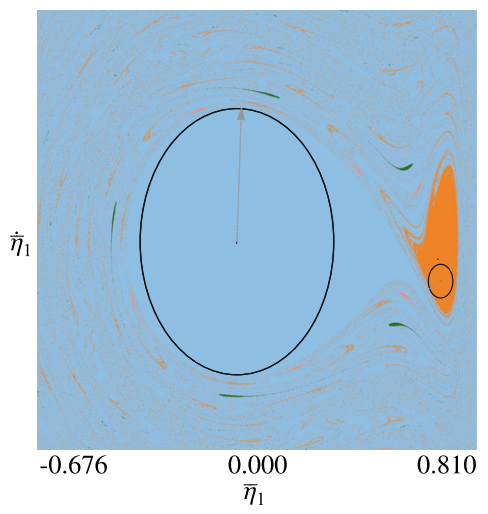

(i) $\bar{y}=0.005, \bar{\Omega}=3.4$
Fig. 11 Variation of basin portraits as a function of excitation frequency $(\bar{\Omega})$ for fixed parameter values of $\bar{y}=0.005$, $\bar{y}=0.020$ and $R=150 \mathrm{~nm}$. The analysis is focused around the fundamental resonance frequencies of respective system attractors. The circle inside the basin portrait indicates the LIM. a-c Basins portraits at $\bar{y}=0.005$ and specific $\bar{\Omega}$ values of $0.6,0.8$,
0.9 , respectively. $\mathbf{d}-\mathbf{f}$ Basins portraits at $\bar{y}=0.020$ and specific $\bar{\Omega}$ values of $0.7,1.8,1.9$, respectively. $\mathbf{g}-\mathbf{i}$ Basins portraits at $\bar{y}=0.005$ and specific $\bar{\Omega}$ values of $2.5,2.9,3.4$, respectively. The details on basin colour and the corresponding attractor/solution description are given in Table 4 
disappearance of several rare attractors, together with period-2 responses appearing not only on $\mathrm{HP}_{1}$ solution branch but also on $\mathrm{Su}_{1} \mathrm{HP}_{1}$ superharmonic solution causes the robustness of in-contact attractor to vary rapidly. This behaviour can be observed in the integrity profiles of Fig. 10d, e in the form of sharp peaks and valleys as the excitation amplitude is increased.

At low excitation frequencies $\bar{\Omega}<1.5$, the incontact basin is dominated by $\mathrm{LP}_{1}$ solution as shown in Fig. 10c. This is further demonstrated in the basin portrait of Fig. 11a where only the light brown basin dominates the in-contact region. Further increasing $\bar{\Omega}>1.5$, we observe the drop in integrity of $\mathrm{LP}_{1}$ solution due to the appearance of $\mathrm{Su}_{1} \mathrm{HP}_{1}$ attractor. This is visualized by comparing Fig. 10c, d between frequency ranges $\bar{\Omega} \in[1,2]$. The drop in integrity is sharp for small values of $\bar{y}$ and slowly smoothens with increasing $\bar{y}$ amplitudes. This effect is due to the increased softening effect of the in-contact response which causes the $\mathrm{Su}_{1} \mathrm{HP}_{1}$ branch to overlap with $\mathrm{LP}_{1}$ branch over larger $\bar{\Omega}$ values. Along with the increased overlapping effect, at higher amplitudes of excitation $\bar{y}>0.00118$, the $\mathrm{Su}_{1} \mathrm{HP}_{1}$ solution grows more robust. The appearance of $\mathrm{Su}_{1} \mathrm{HP}_{1}$ attractor (green basin) from within the $\mathrm{LP}_{1}$ basin for $\bar{y}=0.020$ is shown in Fig. 11e, f.

Furthermore, with $\bar{\Omega}$ growing closer to resonance (i.e. $\bar{\Omega}=3.72$ ), the high-amplitude resonant solution $\left(\mathrm{HP}_{1}\right)$ appears via saddle-node bifurcation and grows in dominance as depicted in Fig. 10e. In a similar fashion to the attractive regime, the saddle-node triggers the erosion of the previously dominant $\mathrm{Su}_{1} \mathrm{HP}_{1}$ solution from outside the boundary. This is observed in basin portraits from Fig. 11g-i where the orange basin belonging to $\mathrm{HP}_{1}$ solution is growing along the periphery of green basin. Interestingly, at low amplitudes of excitation, $\bar{y}<0.010$ the erosion of $\mathrm{Su}_{1} \mathrm{HP}_{1}$ basin is smooth with no influence of rare attractors on its robustness. After the complete erosion of $\mathrm{Su}_{1} \mathrm{HP}_{1}$ basin (see Fig. 11i), the in-contact response is completely dominated by $\mathrm{HP}_{1}$ solution until $\bar{\Omega}$ reaches close to parametric resonance frequency. This is illustrated by the gradual drop in robustness measure in Fig. 10e for values of $\bar{\Omega}>3.7$. The increase in $\bar{y}$ has a peculiar effect on the $\mathrm{HP}_{1}$ solution since the robustness decreases in contrast to the expected increasing trend. This is due to the large number of rare attractors which tend to appear at higher excitation amplitudes. This peculiarity makes the experimental investigation of the in-contact attractor a challenging task.

\subsubsection{Analysis of basin erosion profiles around the principal parametric resonance frequency}

The aforementioned discussion was focused on the robustness and erosion profiles of system attractors for excitation amplitudes below the parametric threshold and excitation frequencies around the fundamental resonance. However, the system excited parametrically exhibits different dynamics that are not observed through direct excitation. In addition, the theoretical analysis of parametrically driven AFM has shown the added benefits such as high quality factor, lower imaging forces and reduced cantilever transients [33]. These advantages, if harnessed, can be of significant interest in areas such as soft polymers and biological specimens. Therefore, the current section focuses on the basin erosion profiles of system attractors in the neighbourhood of respective principal parametric resonance frequencies. Similar to the previous section, the analysis utilizes integrity measures illustrated in Fig. 10 to discuss the evolution of system responses, whereas Fig. 12 is used to understand the metamorphoses of basin erosion graphically.

Considering the attractive force regime, an excitation amplitude of $\bar{y}=0.020$ is utilized to study the robustness of attractors oscillating near the parametric resonance frequency. The chosen excitation amplitude is above the required threshold amplitude of $\bar{y}=0.01515$ needed to excite the system parametrically. The solution belonging to $\bar{y}=0.020$ case is drawn by dotted lines in Fig. 10a. From Fig. 10a there is a sudden decline in the A-HP 1 attractor's integrity value around the parametric resonance frequency of $\bar{\Omega} \in[1.4,1.75]$. This sudden decrease in robustness is promoted by the appearance of period- 2 attractor (A$\mathrm{Pa}-\mathrm{P}_{2}$ ) within the compact part of $\mathrm{A}-\mathrm{HP}_{1}$ basin. This is illustrated in the basin portraits of Fig. 12a-c, where the A-Pa- $\mathrm{P}_{2}$ attractors shown by dark red basin are surrounding the $\mathrm{A}-\mathrm{HP}_{1}$ blue basin. The rate of erosion of $\mathrm{A}-\mathrm{HP}_{1}$ basin is directly proportional to the nearness of $\bar{\Omega}$ to the principal parametric resonance frequency. For values of $\bar{\Omega}$ away from the parametric resonance the A-HP ${ }_{1}$ integrity shows a steady increase back to its original value. Hereafter, the $\mathrm{A}-\mathrm{HP}_{1}$ solution remains almost independent of $\bar{\Omega}$ until the in-contact resonance frequency is reached.

In case of the in-contact regime, the parametric threshold amplitude is found to be as low as $\bar{y}=$ 0.00014. The low threshold amplitude also suggests 


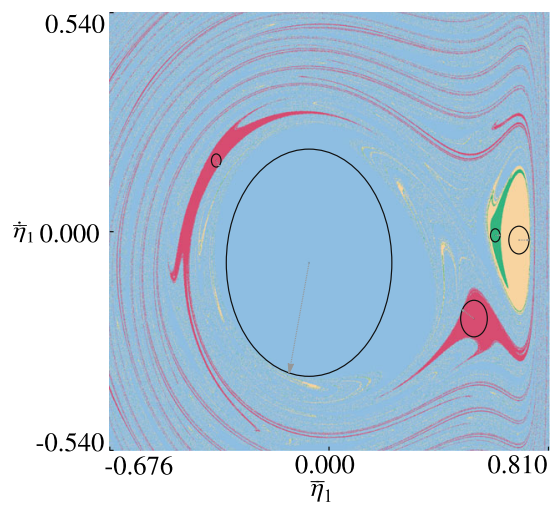

(a) $\bar{y}=0.020, \bar{\Omega}=1.4$

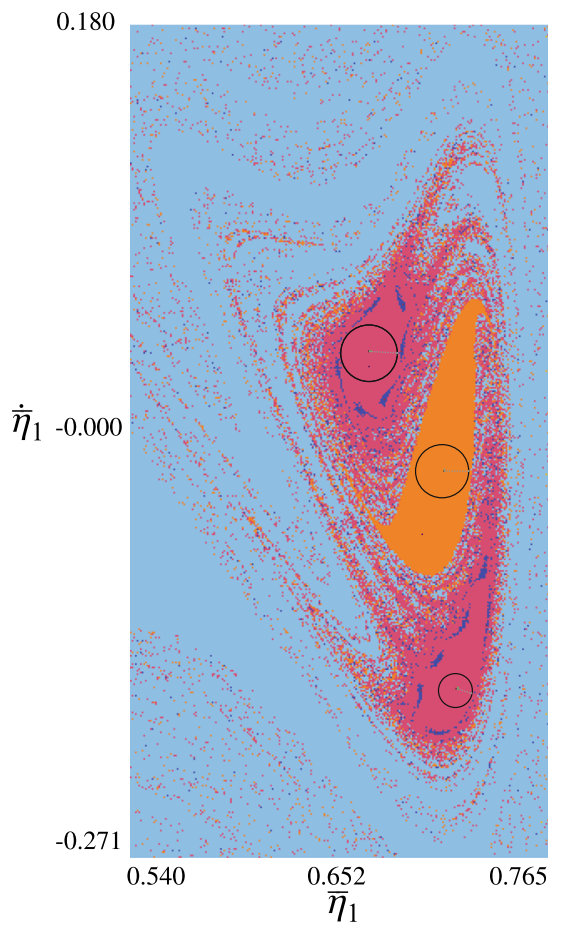

(d) $\bar{y}=0.005, \bar{\Omega}=6.6$

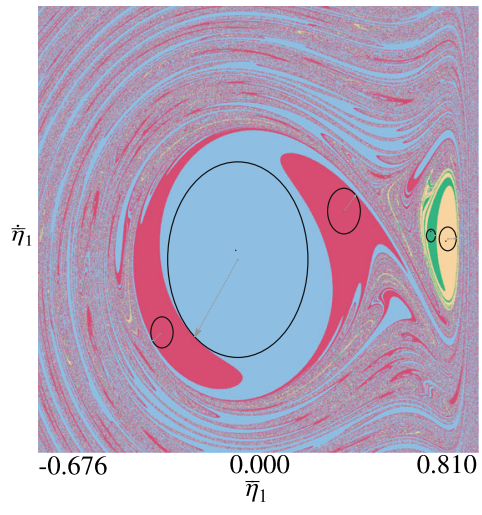

(b) $\bar{y}=0.020, \bar{\Omega}=1.6$

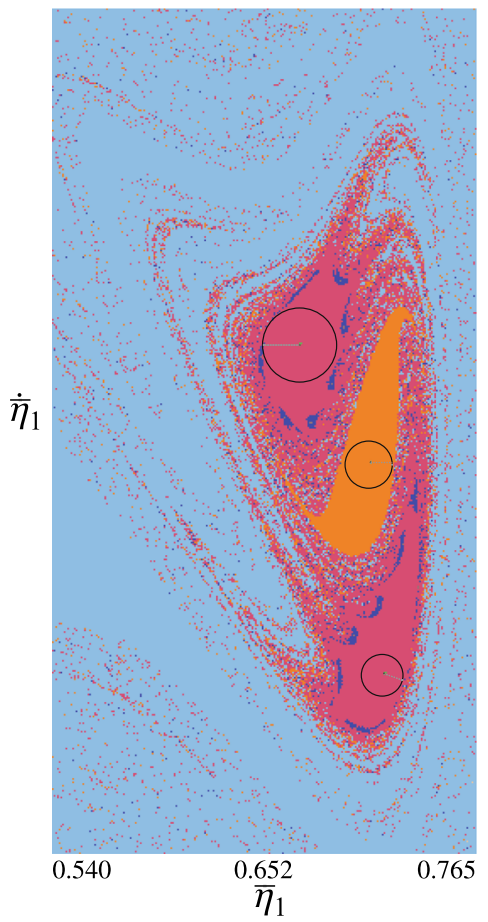

(e) $\bar{y}=0.005, \bar{\Omega}=6.7$

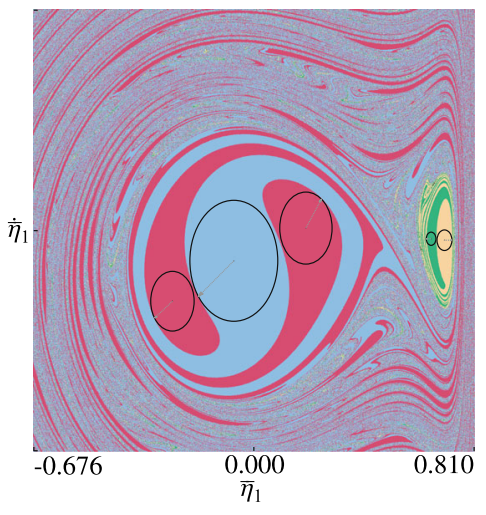

(c) $\bar{y}=0.020, \bar{\Omega}=1.7$

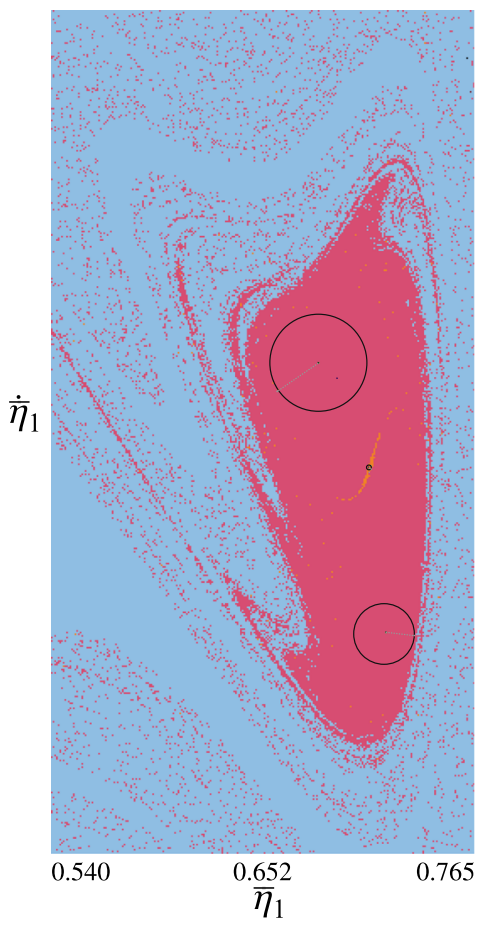

(f) $\bar{y}=0.005, \bar{\Omega}=7.1$
Fig. 12 Variation of basin portraits as a function of excitation frequency $(\bar{\Omega})$ for fixed parameter values of $\bar{y}=0.005$, $\bar{y}=0.020$ and $R=150 \mathrm{~nm}$. The analysis is focused around the principal parametric resonance frequencies of respective system attractors. The circle inside the basin portrait indicates the LIM.

the potential application of stochastic resonance of cantilever oscillating in the in-contact regime. However, in order to facilitate the study, an excitation amplitude of $\bar{y}=0.005$ is utilized. The corresponding integrity profile is illustrated in Fig. 10e by the continuous line, and the relative basin portraits are shown in Fig. 12d, e. By a-c Basin portraits at $\bar{y}=0.020$ and specific $\bar{\Omega}$ values of 1.4 , $1.6,1.7$, respectively. $\mathbf{d}-\mathbf{f}$ Basin portraits at $\bar{y}=0.005$ and specific $\bar{\Omega}$ values of 6.6, 6.7, 7.1, respectively. The details on basin colour and the corresponding attractor/solution description are given in Table 4

considering Fig. 10e, the integrity measure of $\mathrm{HP}_{1}$ solution displays a steady decline as $\bar{\Omega}$ is brought close to principal parametric resonance frequency $(\bar{\Omega}=7.44)$. Finally, around $\bar{\Omega}=7.1$ the $\mathrm{HP}_{1}$ basin completely disappears, indicating that the system is in the parametric instability region. The appearance of paramet- 
ric period-2 attractors $\left(\mathrm{Pa}-\mathrm{P}_{2}\right)$ in the basin portrait is indicated by the dark red basin in Fig. 12d, e. The figures illustrates the appearance of period-2 attractors (dark red) from the compact part of $\mathrm{HP}_{1}$ basin (orange) along with period-6 rare attractors (dark blue). Finally, the erosion process of $\mathrm{HP}_{1}$ solution is non-smooth with the interacting basins featuring severe fractality.

\section{Conclusions}

The global dynamics of TM-AFM has been investigated with the aim of evaluating the robustness and dynamical integrity of coexisting attractors of the system. Extensive numerical analyses have been carried out to show the existence and the properties of the incontact attractor. The frequency response curves and basin portraits are obtained, showing that the in-contact attractor is highly sensitive to the main driven parameters with large amplitude superharmonic branches appearing for small excitation amplitudes.

In order to unveil the entire bifurcation scenario of the so-called in-contact attractor around the primary and parametric resonance, several local bifurcation envelopes are combined together to build global bifurcation maps. Utilizing the bifurcation maps, the escape thresholds along with various response scenarios in the excitation parameter space are analysed in detail around the direct and parametric resonance frequency. The outcome of the analysis shows new routes to crisis, escape scenarios via appearance of strange attractors and multiple period-doubling cascades. In addition, the robustness of attractors has been analysed by making use of basins of attraction and integrity measures such as local integrity measure (LIM) and integrity factor (IF). The analysis has focused on the basin erosion with respect to variation in excitation frequency, excitation amplitude and the AFM probe tip radius. The results highlight the appearance of in-contact attractor for a critical radius value. In addition, the parametric resonance and its effect on basin erosion via fractalization are discussed for both attractive and in-contact attractors. It is seen that the period-2 attractors arising from parametric resonance decrease the robustness of attractive regime in a smooth fashion, whereas in case of in-contact attractor the period- 2 solution together with higher-order strange attractors, erodes the basin through fractalization. In conclusion, our analysis of basins of attraction, global bifurcation charts and integrity profiles provides a method to study the complex dynamics involved in TM-AFM.

Acknowledgements This work is part of the research programme 'NICE TIP TAP' with Grant Number 15450 which is financed by the Netherlands Organisation for Scientific Research (NWO).

\section{Compliance with ethical standards}

Conflict of interest The authors declare that they have no conflict of interest.

Open Access This article is distributed under the terms of the Creative Commons Attribution 4.0 International License (http:// creativecommons.org/licenses/by/4.0/), which permits unrestricted use, distribution, and reproduction in any medium, provided you give appropriate credit to the original author(s) and the source, provide a link to the Creative Commons license, and indicate if changes were made.

\section{Appendix A: Dimensionless variables and coeffi- cients of equation of motion}

The non-dimensional parameters and the corresponding coefficients of Eq. (4) are described below. For further details, the reader is suggested to read the article [12].

$$
\begin{aligned}
\bar{\eta}_{1} & =\frac{x_{1}(\tau)}{\eta^{*}}, x_{1}(\tau)=\phi_{1}(L) q_{1}(\tau), \eta^{*}=Z-w^{*}(L), \\
\tau & =\omega_{1} t, \quad \bar{\Omega}=\frac{\Omega}{\omega_{1}}, \quad d_{1}=\frac{c_{1}}{\omega_{1} \rho A \int_{0}^{L} \phi_{1}^{2} \mathrm{~d} x}, \\
B_{1} & =\left(1-\frac{Z}{\eta^{*}}\right) \Gamma_{1}, \quad C_{11}=-\frac{A_{1} R}{180 k\left(\eta^{*}\right)^{9}} \Gamma_{1}, \\
C_{12} & =-\frac{A_{2} R}{6 k\left(\eta^{*}\right)^{3}} \Gamma_{1}, \quad \omega_{1}^{2}=\frac{E I \int_{0}^{L} \phi_{1} \phi_{1}^{\prime \prime \prime} \mathrm{d} x}{\rho A \int_{0}^{L} \phi_{1}^{2} \mathrm{~d} x}, \\
\Gamma_{1} & =\frac{k \phi_{1}^{2}(L)}{\omega_{1} \rho A \int_{0}^{L} \phi_{1}^{2} \mathrm{~d} x}, \quad k=\frac{3 E I}{L^{3}}, \quad \bar{y}=\frac{Y}{\eta^{*}}, \\
E_{1} & =\frac{\phi(L) \int_{0}^{L} \phi_{1} \mathrm{~d} x}{\int_{0}^{L} \phi_{1}^{2} \mathrm{~d} x} .
\end{aligned}
$$

\section{Appendix B: Instructions on reading the bifurcation chart of Fig. 6}

The instructions on reading the complex bifurcation and response scenarios showcased in Fig. 6 are explained below with the help of a frequency response curve described in Fig. 5. In order to assist with easier 
understanding, the aforementioned figures are reintroduced in this section and marked with coloured circles in Fig. 13 and coloured lines in Fig. 14 at specific $\bar{\Omega}$ values. These coloured lines depict regions of interest. Furthermore, Table 5 delineates the various dynamic response regions seen in Fig. 14.

Referring to Fig. 13, the blue lines which represent the saddle-node bifurcation (SN) loci divide the bifurcation chart into several regions of period-1 solutions such as R1, R2, R4, R5, R6, R7, and R9. Furthermore, the aforementioned saddle-node loci meet at specific values of excitation parameters in pairs of two and give rise to new multi-stable regions at $\bar{\Omega}=1.24$ (intersection of R5 and R10), $\bar{\Omega}=1.851$ (intersection of R2 and $\mathrm{R} 4$ ), and $\bar{\Omega}=3.721$ (intersection of R1 and R9). The dynamic behaviour of system changes depending on whether the excitation amplitude is above or below the point of intersection of saddle-node loci. For instance, in Fig. 13 at $\bar{\Omega}=1.851$ region $\mathrm{R} 2$ exists above and region $\mathrm{R} 4$ below the intersection point.

In particular, special attention should be given to regions where period- 2 solution exists. These regions are bounded by red and green loci corresponding

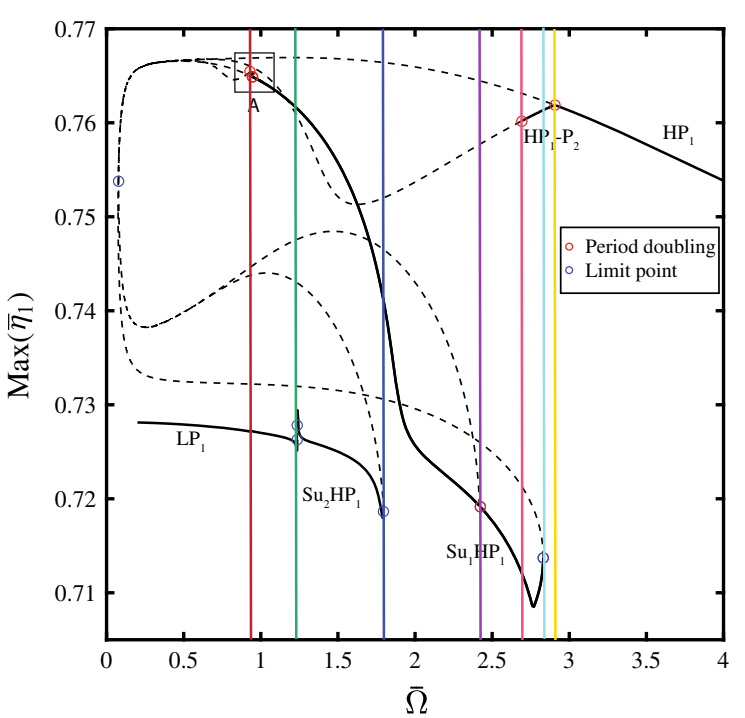

Fig. 14 Frequency response curve of the in-contact attractor for fixed parameter values $\bar{y}=0.020$ and $R=150 \mathrm{~nm}$. Continuous and dotted lines indicate stable and unstable branches of the solution, respectively. Red and blue circles indicate period-doubling and saddle-node bifurcation points, respectively. The coloured vertical lines depict the regions of interest. (Color figure online)

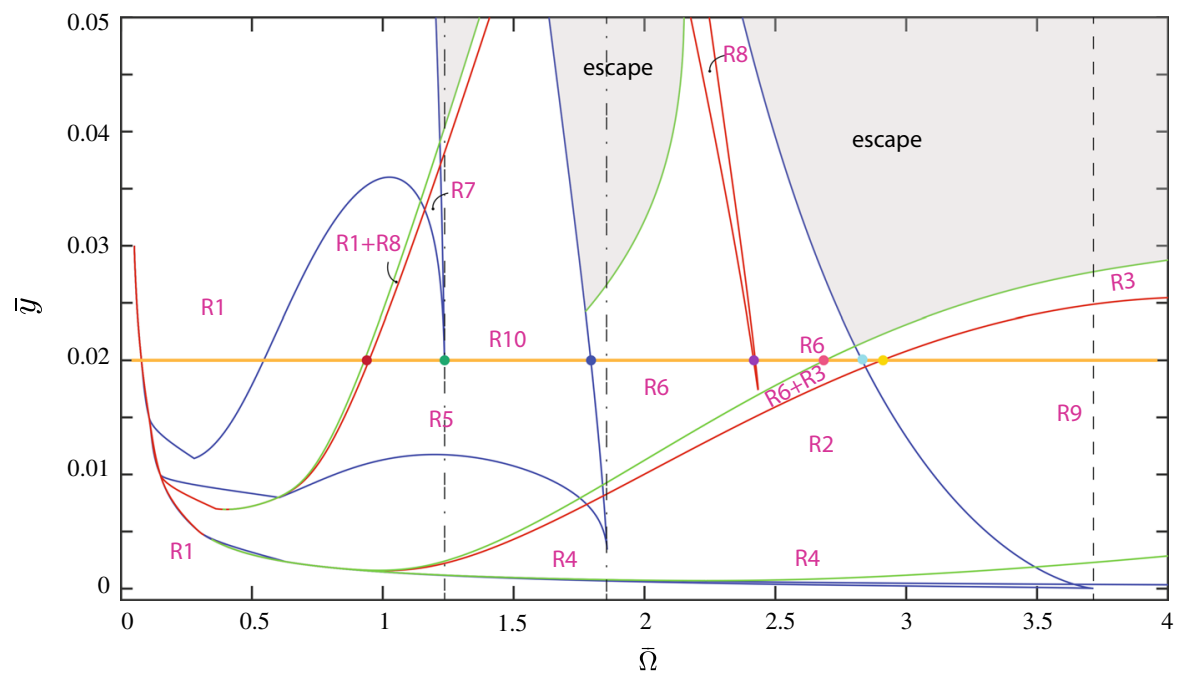

Fig. 13 In-contact attractor bifurcation and response chart focusing on the region below the primary resonance frequency $\left(\bar{\Omega}_{0}=3.721\right.$ indicated by the vertical dashed line). The first and second superharmonic frequencies are indicated at $\bar{\Omega}=1.851$ and $\bar{\Omega}=1.24$ by dashed-dotted lines. Blue lines are the saddlenode bifurcation loci on period-1 solution branches, red lines are the period-doubling/flip bifurcation loci on period-1 solution branches, and green lines are the period-doubling/flip bifurca- tion loci on period-2 solution branches. The details on individual bifurcation envelope description and the corresponding solution regions are summarized in Tables 2 and 3, respectively. The light brown horizontal line at $\bar{y}=0.020$ is provided for easy correlation of data. The coloured circles on the horizontal line mark the corresponding regions of interest as indicated in Fig. 14. (Color figure online) 
Table 5 Correlation between the regions and solutions in Fig. 13 and corresponding coloured vertical lines in Fig. 14

\begin{tabular}{lll}
\hline $\begin{array}{l}\text { Line color } \\
\text { start } \rightarrow \text { end })\end{array}$ & $\begin{array}{l}\text { Dynamic region } \\
(\text { start } \rightarrow \text { end })\end{array}$ & $\begin{array}{l}\text { Solution branch } \\
(\text { start } \rightarrow \text { end })\end{array}$ \\
\hline$\rightarrow \mid$ & $\mathrm{R} 1 \rightarrow(\mathrm{R} 1+\mathrm{R} 8)$ & $\mathrm{LP}_{1} \rightarrow\left(\mathrm{LP}_{1}+\mathrm{Su}_{1}-\mathrm{P}_{2}\right)$ \\
$\mid$ & $\mathrm{R} 5 \rightarrow \mathrm{R} 7$ & $\begin{array}{l}\left(\mathrm{LP}_{1}+\mathrm{Su}_{1} \mathrm{HP}_{1}\right) \rightarrow \\
\left(\mathrm{LP}_{1}+\mathrm{Su}_{1} \mathrm{HP}_{1}+\mathrm{Su}_{2} \mathrm{HP}_{1}\right)\end{array}$ \\
$\mid$ & $\mathrm{R} 10 \rightarrow \mathrm{R} 6$ & $\left(\mathrm{Su}_{1} \mathrm{HP}_{1}+\mathrm{Su}_{2} \mathrm{HP}_{1}\right)$ \\
$\mid$ & $\mathrm{R} 6 \rightarrow \mathrm{R} 8$ & $\mathrm{Su}_{1} \mathrm{HP}_{1} \rightarrow\left(\mathrm{Su}_{1}-\mathrm{P}_{2}\right)$ \\
$\mid$ & $\mathrm{R} 6 \rightarrow(\mathrm{R} 6+\mathrm{R} 3)$ & $\mathrm{Su}_{1} \mathrm{HP}_{1} \rightarrow\left(\mathrm{Su}_{1} \mathrm{HP}_{1}+\mathrm{Su}_{1}-\mathrm{P}_{2}\right)$ \\
$\mid$ & $(\mathrm{R} 6+\mathrm{R} 3) \rightarrow \mathrm{R} 3$ & $\left(\mathrm{Su}_{1} \mathrm{HP}_{1}+\mathrm{HP}_{1}-\mathrm{P}_{2}\right) \rightarrow \mathrm{HP}_{1}-\mathrm{P}_{2}$ \\
$\mid$ & $\mathrm{R} 3 \rightarrow \mathrm{R} 9$ & $\mathrm{HP}_{1}-\mathrm{P}_{2} \rightarrow \mathrm{HP}_{1}$ \\
& & \\
\hline
\end{tabular}

to period-doubling/flip bifurcations. In general the period-2 solutions are bound to narrow regions over a small range of excitation parameters (except for parametric resonance case). These narrow regions invade into larger regions of period-1 solutions resulting in new intersection regions where combination of period1 and period- 2 solutions exists. An example of such an intersecting region is indicated in Fig. 13 as R6 + R3 where $\mathrm{HP}_{1}-\mathrm{P}_{2}$ and $\mathrm{Su}_{1} \mathrm{HP}_{1}$ solutions coexist. Finally, by utilizing the aforementioned instructions the bifurcation behaviour observed in Fig. 5 is described below.

An excitation parameter equal to $\bar{y}=0.020$ is utilized in simulating Fig. 14. This value is further highlighted in Fig. 13 with a light brown horizontal line spanning across the entire range of the excitation frequency. Referring to Fig. 14, the first region of interest is marked by a red vertical line and spans region up to $\bar{\Omega} \leq 0.93$. From Fig. 13, this region starts with $\mathrm{LP}_{1}$ solution marked by $\mathrm{R} 1$ and ends with $\mathrm{LP}_{1}+\mathrm{Su}_{1}-\mathrm{P}_{2}(\mathrm{R} 1+\mathrm{R} 8)$ solution at $\bar{\Omega}=0.93$. The second region of interest is between the red and green vertical lines in Fig. 14 and ranges from $\bar{\Omega} \in$ $(0.93,1.236]$. From Fig. 13 , this region starts with $\mathrm{LP}_{1}$ $+\mathrm{Su}_{1} \mathrm{HP}_{1}$ solution indicated by $\mathrm{R} 5$ from $\bar{\Omega}>0.93$ and ends with multi-stable region $\mathrm{R} 7$ consisting of $\mathrm{LP}_{1}+\mathrm{Su}_{1} \mathrm{HP}_{1}+\mathrm{Su}_{2} \mathrm{HP}_{1}$ solutions at $\bar{\Omega}=1.236$. Similarly, the third region can be found between the green and blue vertical lines in Fig. 14 and ranges from
$\bar{\Omega} \in(1.236,1.795]$. This region shows the presence of $\mathrm{Su}_{1} \mathrm{HP}_{1}+\mathrm{Su}_{2} \mathrm{HP}_{1}$ solutions marked by R10 from $\bar{\Omega}>1.236$ up to $\bar{\Omega}=1.795$. The next region of interest is bounded by blue and purple vertical lines in Fig. 14 and ranges from $\bar{\Omega} \in(1.795,2.413]$. Referring to Fig. 13 , for $\bar{\Omega}>1.795$, the region exhibits monostability consisting of only $\mathrm{Su}_{1} \mathrm{HP}_{1}$ solution marked by region $\mathrm{R} 6$ and ends with region $\mathrm{R} 8$ consisting of $\mathrm{Su}_{1}$ $\mathrm{P}_{2}$ solution at $\bar{\Omega}=2.413$. Continuing along the same trend, the other sections highlighted in Fig. 14 can be traced and are shown in Table 5.

\section{References}

1. Gross, L., Mohn, F., Moll, N., Liljeroth, P., Meyer, G.: The chemical structure of a molecule resolved by atomic force microscopy. Science 325(5944), 1110-1114 (2009)

2. Zhong, Q., Inniss, D., Kjoller, K., Elings, V.B.: Fractured polymer/silica fiber surface studied by tapping mode atomic force microscopy. Surf. Sci. Lett. 290(1), L688-L692 (1993)

3. Garcia, R.: Theory of Amplitude Modulation AFM. WileyVCH Verlag GmbH \& Co. KGaA, Weinheim (2010)

4. Paulo, ASan, Garcia, R.: High-resolution imaging of antibodies by tapping-mode atomic force microscopy: attractive and repulsive tip-sample interaction regimes. Biophys. J. 78(3), 1599-1605 (2000)

5. Stark, M., Moeller, C., Mueller, D.J., Guckenberger, R.: From images to interactions: high-resolution phase imaging in tapping-mode atomic force microscopy. Biophys. J. 80(6), 3009-3018 (2001)

6. Knoll, A., Magerle, R., Krausch, G.: Tapping mode atomic force microscopy on polymers: where is the true sample surface? Macromolecules 34(12), 4159-4165 (2001)

7. Sarid, D.: Scanning Force Microscopy with Applications to Electric, Magnetic and Atomic Forces, vol. 14. Oxford University Press, Oxford (1991)

8. Hoelscher, H., Allers, W., Schwarz, U.D., Schwarz, A., Wiesendanger, R.: Determination of tip-sample interaction potentials by dynamic force spectroscopy. Phys. Rev. Lett. 83(23), 4780-4783 (1999)

9. Raman, A., Trigueros, S., Cartagena, A., Stevenson, A.P., Susilo, M., Nauman, E., Contera, S.A.: Mapping nanomechanical properties of live cells using multi-harmonic atomic force microscopy. Nat. Nanotechnol. 6(12), 809-14 (2011)

10. Trinidad, E.Rull, Gribnau, T.W., Belardinelli, P., Staufer, U., Alijani, F.: Nonlinear dynamics for estimating the tip radius in atomic force microscopy. Appl. Phys. Lett. 111(12), 123105 (2017)

11. Garcia, R., Paulo, ASan: Dynamics of a vibrating tip near or in intermittent contact with a surface. Phys. Rev. B 61(20), R13381-R13384 (2000)

12. Ruetzel, S., Lee, A., Raman, Sand: Nonlinear dynamics of atomic force microscope probes driven in Lennard-Jones potentials. Proc. R. Soc. Lond. Ser. A Math. Phys. Eng. Sci. 459(2036), 1925-1948 (2003) 
13. Lee, S.I., Howell, S.W., Raman, A., Reifenberger, R.: Nonlinear dynamic perspectives on dynamic force microscopy. Ultramicroscopy 97(1-4), 185-198 (2003)

14. Bahrami, A., Nayfeh, A.H.: On the dynamics of tapping mode atomic force microscope probes. Nonlinear Dyn. 70(2), 1605-1617 (2012)

15. Wolf, K., Gottlieb, O.: Nonlinear dynamics of a noncontacting atomic force microscope cantilever actuated by a piezoelectric layer. J. Appl. Phys. 91(7), 4701-4709 (2002)

16. Garcia, R., Paulo, ASan: Attractive and repulsive tipsample interaction regimes in tapping-mode atomic force microscopy. Phys. Rev. B 60(7), 4961-4967 (1999)

17. Paulo, A.S., Garcia, R.: Tip-surface forces, amplitude, and energy dissipation in amplitude-modulation (tapping mode) force microscopy. Phys. Rev. B 64(19), 193411 (2001)

18. Hashemi, N., Dankowicz, H., Paul, M.R.: The nonlinear dynamics of tapping mode atomic force microscopy with capillary force interactions. J. Appl. Phys. 103(9), 093512 (2008)

19. Rega, G., Settimi, V.: Bifurcation, response scenarios and dynamic integrity in a single-mode model of noncontact atomic force microscopy. Nonlinear Dyn. 73(1), 101-123 (2013)

20. Hornstein, S., Gottlieb, O.: Nonlinear dynamics, stability and control of the scan process in noncontacting atomic force microscopy. Nonlinear Dyn. 54(1-2), 93-122 (2008)

21. Settimi, V., Rega, G.: Global dynamics and integrity in noncontacting atomic force microscopy with feedback control. Nonlinear Dyn. 86(4), 2261-2277 (2016)

22. Israelachvili, J.N.: Intermolecular and Surface Forces, 3rd edn. Academic Press, San Diego (2011)

23. Ulcinas, A., Snitka, V.: Intermittent contact afm using the higher modes of weak cantilever. Ultramicroscopy 86(1), 217-222 (2001)

24. Eusebius, J.D., Alan, R.C., Thomas, F.F., Yuri, A.K., Bjoern, S., Xianjun, W.: AUTO-07P: Continuation and bifurcation software for ordinary differential equations (1998)
25. Keyvani, A., Sadeghian, H., Goosen, H., van Keulen, F.: On the origin of amplitude reduction mechanism in tapping mode atomic force microscopy. Appl. Phys. Lett. 112(16), 163104 (2018)

26. Keyvani, A., Alijani, F., Sadeghian, H., Maturova, K., Goosen, H., van Keulen, F.: Chaos: the speed limiting phenomenon in dynamic atomic force microscopy. J. Appl. Phys. 122(22), 224306 (2017)

27. Soliman, M.S., Thompson, J.M.T.: Integrity measures quantifying the erosion of smooth and fractal basins of attraction. J. Sound Vib. 135(3), 453-475 (1989)

28. Belardinelli, P., Lenci, S.: An efficient parallel implementation of cell mapping methods for mdof systems. Nonlinear Dyn. 86(4), 2279-2290 (2016)

29. Belardinelli, P., Lenci, S.: A first parallel programming approach in basins of attraction computation. Int. J. Non Linear Mech. 80, 76-81 (2016)

30. Belardinelli, P., Lenci, S., Rega, G.: Seamless variation of isometric and anisometric dynamical integrity measures in basins erosion. Commun. Nonlinear Sci. Numer. Simul. 56, 499-507 (2018)

31. Lenci, S., Rega, G.: Optimal control of nonregular dynamics in a duffing oscillator. Nonlinear Dyn. 33(1), 71-86 (2003)

32. Vahdat, V., Carpick, R.W.: Practical method to limit tip sample contact stress and prevent wear in amplitude modulation atomic force microscopy. ACS Nano 7(11), 9836-9850 (2013)

33. Prakash, G., Hu, S., Raman, A., Reifenberger, R.: Theoretical basis of parametric-resonance-based atomic force microscopy. Phys. Rev. B 79(9), 094304 (2009)

Publisher's Note Springer Nature remains neutral with regard to jurisdictional claims in published maps and institutional affiliations. 